\title{
A review of liquid flow and heat transfer in microchannels with emphasis to electronic cooling
}

\author{
APARESH DATTA ${ }^{1}$, DIPANKAR SANYAL ${ }^{2}$, AMIT AGRAWAL $^{3, *}$ and AJOY KUMAR DAS ${ }^{4}$ \\ ${ }^{1}$ Department of Mechanical Engineering, National Institute of Technology Durgapur, Durgapur 713209, India \\ ${ }^{2}$ Department of Mechanical Engineering, Jadavpur University, Kolkata 700032, India \\ ${ }^{3}$ Department of Mechanical Engineering, Indian Institute of Technology Bombay, Powai, Mumbai 400076, \\ India \\ ${ }^{4}$ Department of Mechanical Engineering, National Institute of Technology Agartala, Agartala 799046, India \\ e-mail: amit.agrawal@iitb.ac.in; aagrawal.iitb@gmail.com
}

MS received 1 January 2019; revised 19 June 2019; accepted 26 August 2019

\begin{abstract}
Since the realization of microchannel devices more than three and half decades ago with water as the cooling fluid providing heat transfer enhancement, significant progress has been made to improve the cooling performance. Thermal management for electronic devices with their ever-widening user profile remains the major driving force for performance improvement in terms of miniaturisation, long-term reliability, and ease of maintenance. The ever-increasing requirement of meeting higher heat flux density in more compact and powerful electronic systems calls for further innovative solutions. Some recent studies indicate the promise offered by processes with phase change and the use of active devices. But their adoption for electronic cooling still weighs unfavourably against long-term fluid stability and simplicity of device profile with moderate to high heat transfer capability. Applications and reviews of these promising research trends have been briefly visited in this work. The main focus of this review is the flow and heat transfer regime related to electronic cooling in evolving channel forms, whose fabrication are being enabled by the significant advancement in micro-technologies. Use of disruptive wall structures like ribs, cavities, dimples, protrusions, secondary channels and other interrupts along with smooth-walled channels with curved flow passages remain the two chief geometrical innovations envisaged for these applications. These innovations target higher thermal enhancement factor since this implies more heat transfer capability for the same pumping power in comparison with the corresponding straight-axis, smooth-wall channel configuration. The sophistication necessary to deal with the experimental uncertainties associated with the micron-level characteristic length scale of any microchannel device delayed the availability of results that exhibited acceptable matching with numerical investigations. It is indeed encouraging that the experimental results pertaining to simple smooth channels to grooved, ribbed and curved microchannels without unreasonable increase in pumping power have shown good agreement with conventional numerical analyses based on laminar-flow conjugate heat-transfer model with no-slip boundary condition. The flow mechanism with the different disruptive structures like dimple, cavity and rib, fin and interruption, vortex generator, convergingdiverging side walls or curved axis are reviewed to augment the heat transfer. While the disruptions cause heat transfer enhancement by interrupting the boundary layer growth and promoting mixing by the shed vortices or secondary channel flow, the flow curvature brings in enhancement by the formation of secondary rolls culminating into chaotic advection at higher Reynolds number. Besides these revelations, the numerical studies helped in identifying the parameter ranges, promoting a particular enhancement mechanism. Also, the use of modern tools like Poincare section and the analysis of flow bifurcation leading to chaotic advection is discussed. Among the different disruptive structures, sidewall cavity with rib on the bottom wall within the cavity plays a significant role in augmenting the thermal performance. Among the different converging-diverging side walls or curved axis, the sinusoidal channel provides the highest mixing by the introduction of secondary vortices or dean vortices to augment the heat transfer with less pressure drop. The optimum geometry in terms of high heat transfer with low pressure plays a major role in the design of heat sink. Directions of some future research are provided at the end.
\end{abstract}

Keywords. Thermal enhancement factor; vortex generator; grooved, rib and curved microchannels.

*For correspondence

Published online: 15 November 2019 


\section{Motivation}

The desire for endowing electronic and mechatronic devices with more compactness, greater functional capabilities and wider usage has been pushing researchers to arrive at cooling solutions with higher power-density, higher portability and easier maintainability. Rapid miniaturization and increased power density of modern electronic devices demand more compact and reliable cooling solutions so as to avoid shortening of the life span of the devices. Existing cooling solutions require further innovations for sustaining the projected cooling demand of sophisticated VLSI devices [1], for instance. According to a 2013 report of International Technology Roadmap for Semiconductors, heat flux of about $100 \mathrm{~W} / \mathrm{cm}^{2}$ is to be removed from a $14 \mathrm{~nm}$ chip [2]. The generation of heat flux by high-power electronic components and laser diodes like GaAs and Si based transistor amplifiers is close to $100-300 \mathrm{~W} / \mathrm{cm}^{2}$ [3]. In near future, the cooling demand of electronic device is expected to go up to $1000 \mathrm{~W} / \mathrm{cm}^{2}$ [4]. Tuckerman and Pease [1] found a contemporary solution of heat transfer enhancement by micromachining an arrangement of straight and parallel rectangular microchannels on a silicon wafer base and using water as the coolant flowing through the channels. Increased surface area provided by the intermediate side walls in a microchannel sink increases the heat transfer with respect to a single-channel sink of same flow volume. Figure 1 describes the core of a microchannel system considered by many earlier researchers in numerical and experimental studies $[5,6]$. The channels in the core zone receive cold fluid from the inlet through an inlet header. As a result of the heat flux from the channel bottom, the fluid becomes hot and leaves the core zone through the outlet header and outlet. The inlet and the outlet are provided on the top plate covering the base. Different header configurations with different inlet-outlet positioning are shown figure 1(c). An experimental set-up should also involve pump connecting the inlet and outlet, a valve in order to achieve different discharges, a heater with a controller for providing different bottom heat flux along with pressure transducers, flow meter and thermocouples for assessing the thermal performance of the heat sink from the measurements.

Over and above the walls at two extreme sides of a single channel, a multiple-channel system has intermediate side walls, as shown in figure 1(a) in a representative manner with rectangular, triangular and trapezoidal channel shapes. Of course, the intermediate walls provide increased surface area for heat transfer from channel bottom to the fluid of similar flow volume in both the cases. However, this increase in wall area also causes the friction factor to be higher. In other words, there arises a concurrent

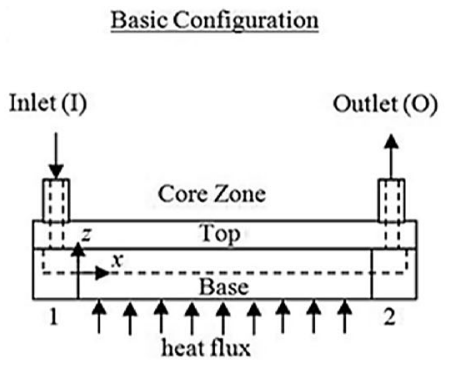

1: Inlet Header $(\mathrm{IH})$

2: Outlet Header $(\mathrm{OH})$

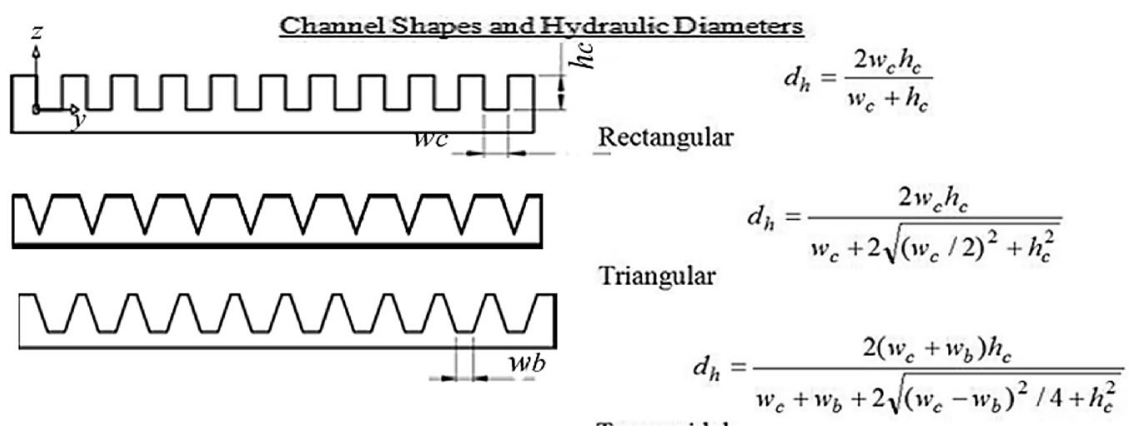

(a)
Header Types

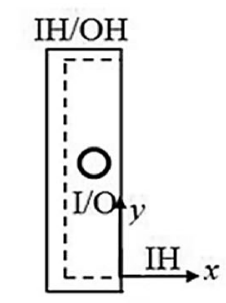

Rectangular
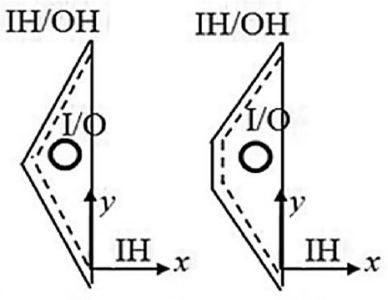

Triangular Trapezoidal

(b)
Types of I/O Connections

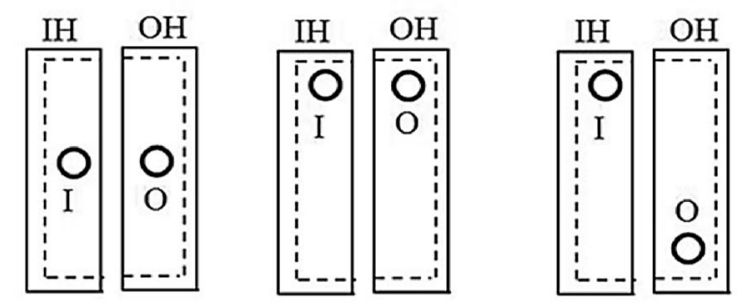

Central or I type One sided or C type Diagonal or $Z$ type

(c)

Figure 1. Smooth and straight microchannel systems. (a) Overall configuration with different channel shapes, (b) different header shapes with central inlet-outlet connections and $(c)$ different inlet-outlet connections in rectangular headers. 
enhancement of both length-averaged wall Nusselt number $\mathrm{Nu}$ and friction factor $\mathrm{f}$ that are the measures of heat transfer and pressure drop, respectively. Results presented by Kandlikar and Grande [6] for fully developed laminar flow under constant wall heat flux condition clearly showed that decrease in hydraulic diameter entails simultaneous increase in both $\mathrm{Nu}$ and $\mathrm{f}$. Microchannels were classified by the hydraulic diameter of the channel ranging between 10 $\mu \mathrm{m}$ and $200 \mu \mathrm{m}$. The classification by Mehendale et al [7] involves the smallest dimension of the channel with the lower bound reduced to $1 \mu \mathrm{m}$. However, such a low size may call for employing slip boundary condition for modeling gas flow. Agrawal et al [8] clearly established the need of incorporating slip boundary condition for modeling gas flow in channels of such low depth.

A major research motivation in the context of microchannel systems is to achieve high $\mathrm{Nu}$ at a reasonable $\mathrm{f}$ by incorporating certain modifications over smooth and straight channels. Any modification would indeed alter both $\mathrm{Nu}$ and $\mathrm{f}$. Webb [9] defined a thermal enhancement factor (TEF) as the ratio of enhanced heat transfer coefficient achieved with identical pumping power in case of the modified channel to that of a corresponding basic channel with smooth wall and straight axis. For channels having the same basic cross sectional area, both the average flow velocity and the discharge are proportional to Reynolds number Re. The well-known Darcy law provides the relation for pressure drop in a channel to be proportional to both friction factor $f$ and square of the velocity. Therefore, the pumping power $\mathrm{P}$, given by the product of the discharge and the pressure drop, can be expressed as proportional to f. $\operatorname{Re}^{3}$. Using 0 as the subscript for the basic channel, it can be written that

$$
\mathrm{P} / \mathrm{P}_{0}=\left(\mathrm{f} \cdot \operatorname{Re}^{3}\right) /\left(\mathrm{f}_{0} \cdot \operatorname{Re}_{0}^{3}\right) \Rightarrow \operatorname{Re}_{0}=\left(\mathrm{f} / \mathrm{f}_{0}\right)^{1 / 3} \operatorname{Re} \text { for } \mathrm{P}=\mathrm{P}_{0} \text {. }
$$

Of course, the $\mathrm{Nu}$ ratio corresponding to the same pumping power implies different Reynolds numbers for the basic and the modified channels, whereas it is customary to define the ratios of Nusselt number and friction factor for constant Re. Assuming a linear relation between $\mathrm{Nu}$ and $\mathrm{Re}$ for not too disparate Re values along with using the above relation for the $\mathrm{Re}$ ratio, the thermal enhancement factor can be obtained as

$$
\begin{aligned}
\mathrm{TEF} & =\left.\left(\mathrm{Nu} / \mathrm{Nu}_{0}\right)\right|_{\mathrm{PP}}=\left.\mathrm{Nu}\right|_{\mathrm{Re}} /\left.\mathrm{Nu}_{0}\right|_{\mathrm{Re}_{0}} \\
& =\left.\mathrm{Nu}\right|_{\operatorname{Re}} /\left\{\left.N u_{0}\right|_{\operatorname{Re}} \times\left(\operatorname{Re} / \operatorname{Re}_{0}\right)\right\} \\
& \Rightarrow \mathrm{TEF}=\left.\left(\mathrm{Nu} / \mathrm{Nu}_{0}\right)\right|_{\mathrm{Re}} /\left\{\left.\left(\mathrm{f} / \mathrm{f}_{0}\right)\right|_{\mathrm{Re}}\right\}^{1 / 3} .
\end{aligned}
$$

As the value of TEF goes more and more above unity, it signifies a higher domination of the gain in the form of heat transfer enhancement over the penalty of the pressure drop incurred. This factor, also referred to as thermal performance, is popularly used to compare the performance enhancement arising from different geometric innovations over smooth and straight channels.
Adham et al [10] concluded from their review that most of the studies prior to year 2000 were experimental in nature, whereas a much larger portion of later research is dominated by conventional laminar-flow simulation with no-slip wall boundary condition and continuum approach for the flow. Kaushik et al [11] reported that the implementation of slip boundary condition results in an increase in $\mathrm{Nu}$. A conjugate heat transfer analysis by Shkarah et al [12] revealed that the thermal resistance of a grapheme substrate is lower than the ones with silicon and aluminum. Such analyses by Hung et al [13, 14] indicated that the demerit of high pumping power requirement for flow through microchannels filled with sintered porous material could be alleviated by providing height or width enlargement to the channels. Numerical results of Farsad et al [15] and Shen et al [16] exhibited a more uniform sink temperature profile provided by microchannels partially filled by porous metal foam. Shen et al [16] validated their model against the results of Calmidi and Mahajan [17] for low Re flow through a continuous foam structure. For Re from 170 to 554 and porosity from 0.7 to 0.9 , they found a mixed arrangement of continuous structure between two Y-shaped foams, one near the inlet and the other near the outlet, to yield higher enhancement of heat transfer in comparison to both the continuous and repeated Y structures. Adham et al [10] provided an account of flow of different coolants such as air, helium, argon, nitrogen, water, deionized water, paraffin, and nanofluids through microchannels made of aluminum, copper, stainless steel, titanium, silicon, bronze, pyrex glass, silica glass, quartz, polyimide and polycarbonate, considered by various researchers. Of course, liquid coolants are more capable than gas or air to fulfil the projected high power-density thermal-management needs.

The ever-increasing demand of the mixing and cooling rates keeps the fluid flow and heat transfer research in microchannel geometry live with a gradual shift from air flow to liquid flow for meeting the cooling demand in the presence of high rate of heat flux. Several modifications in the form of flow disruptions by placing internal structures in the flow passage or by making the flow to follow a tortuous path through a channel with curved or zigzag axis are being investigated. Of course, this research is being constantly supported by the continuous advancement in micro-fabrication technology. Phillips [18] and Kandlikar and Grande [6, 19] discussed the salient aspects of this technology. Dixit and Ghosh [20] reviewed the fabrication processes linked with applications in micro-pump, microturbine, micro-motor, micro-valve, and micro-reactor as heat sink, as well as in gas liquefaction plant, cryosurgical probe, miniature cryocooler, aircraft gas-turbine engine, and spacecraft as miniature heat exchanger. Improvisations like multiphase flow and use of nanofluids have been studied in the context of both unmodified and modified channels. However, the issues of poor long-term stability of nanofluids and increased device complexities for tackling 
multi-phase flow situations keep the research interest live in single-phase microchannel studies.

A rigorous review has been carried out here on singlephase heat transfer augmentation techniques without incurring significant rise in the pressure drop in microchannel. This review consists of three parts. The second section discusses smooth and simple geometrical configuration, and effect of other parameters associated with the geometry. The third section describes channels with different disruptive structures, while the forth section discusses channels with converging-diverging side walls or channels with a curved axis. In each section, some suggestions are included to further augment the TEF of the microsink. Some concluding remarks are provided in the last section.

\section{Smooth and straight channels}

Laminar-flow numerical studies have been carried out through straight microchannels of simple rectangular to more complex geometric shapes, of different aspect ratios (defined as the ratio of width to depth of the channel). Croce and D'Agaro [21] analyzed flow between parallel plates and through circular tube. McHale and Garimella [22] investigated flow through trapezoidal section, whereas Gunnasegaran [23] dealt with rectangular, triangular and trapezoidal sections as described in figure 1. Morini [24, 25] carried out theoretical and experimental investigations with these shapes as well as a hexagonal section. Zhuo et al [26] obtained good agreement of their numerical predictions with existing results and predicted higher $\mathrm{Nu}$ of trapezoidal microchannels over triangular ones. $\mathrm{Wu}$ and Cheng [27] experimentally found that friction factor depends on the ratio of top to the base width of trapezoidal microchannel. McHale and Garimella [22] established that heat transfer depends on the aspect ratio of trapezoidal microchannel. Sadavisam et al [28] found the heat transfer in trapezoidal and hexagonal channels to depend on the side angle and aspect ratio of microchannel. Saha et al [29] found that heat transfer of a rhombic channel depends on its side angle and validated their numerical model against available experimental results. They used the model to develop a correlation for $\mathrm{Nu}$ in terms of the angle of a rhombic flow section. These studies related to different cross-sections revealed no order of magnitude difference in either $\mathrm{Nu}$ or $\mathrm{f}$ for similar Reynolds number defined on the basis of hydraulic diameter $d_{h}$. The expression of the hydraulic diameter in terms of the geometric parameters of each rectangular, triangular or trapezoidal channel sections is provided in figure 1(a) depicting smooth and straight microchannel systems.

Tables 1 and 2 provide a summary of different related studies pertaining to microchannel systems described in figure 1. Correlations of the performance parameters obtained from the experimental studies are listed in the tables, with some symbols explained locally in the table and some in the nomenclature. The predictions of the numerical studies exhibited some deviations from the performance parameters from earlier experimental results by Wang and Peng [30], Peng and Peterson [31] and Qu et al [32] for instance. These studies do not reveal the significance of entrance effect and exit loss. Morini [25], Hestroni et al [33] and Valdes et al [34] mentioned about the error in measuring the channel dimensions and other uncertainties in earlier experiments can be attributed as a significant factor in the observed disagreement. But the deviations prompted computational studies by researchers like Hu et al [35], Rawool et al [36], Croce et al [37] and Gamrat et al [38] along with the experiments by $\mathrm{Wu}$ and Cheng [39] to investigate the effect of surface roughness of different postulated types as given in table 3 .

Croce et al [21, 37], Hu et al [35] and Rawool et al [36] found the size and orientation of conical and rectangular prism-shaped roughness elements to affect the heat transfer and pressure drop in microchannels. An important observation is that if the order is $10^{-5}$, the roughness effect is negligible [39]. Hu et al [35] found that beyond a spacing of $30 \mu \mathrm{m}$ between the roughness elements, the channel behaves like a smooth channel. Hence, it is understandable that later experiments [40-42] exhibited good matching with laminar-flow predictions. Silicon is preferred over aluminium for achieving smoother surface. In case rough channels make the flow turbulent, unacceptably high pressure drop arises without significant augmentation of heat transfer. Hence, turbulence modelling or high Re studies did not receive much attention in numerical investigations in the context of microchannel flow. Major focus of such research was on studying the effects of conjugate heat transfer, thermo-physical property variations of both the solid and the liquid and use of inclined surfaces as vortex generators and discrete disruptive structures like cavities, fins and microchambers.

$\mathrm{Qu}$ and Mudawar [43] performed conjugate heat transfer analysis as well as experiments with applied heat flux of 100 and $200 \mathrm{~W} / \mathrm{cm}^{2}$. They established the applicability of laminar flow model for numerical analysis of single-phase liquid flow through smooth and straight microchannels for $\mathrm{Re}$ in the range of 139 to 1672 . Liu and Garimella [44] observed that conventional correlations provide reliable predictions for laminar flow through rectangular microchannels for hydraulic diameter as low as $250 \mu \mathrm{m}$. Liu et al [45] considered the flow is laminar as the conventional laminar flow. A conjugate heat transfer laminar flow analysis by Mansoor et al [46] under heat flux up to $130 \mathrm{~W} / \mathrm{cm}^{2}$ over Re range from 500 to 2000 yielded $\mathrm{Nu}$ variation within $5 \%$ experimental results of Lee at al [47]. The experimental results of Tiselj et al [48] revealed good matching with their numerical predictions in the $\mathrm{Re}$ range of 3.2-64. Lee and Garimella 


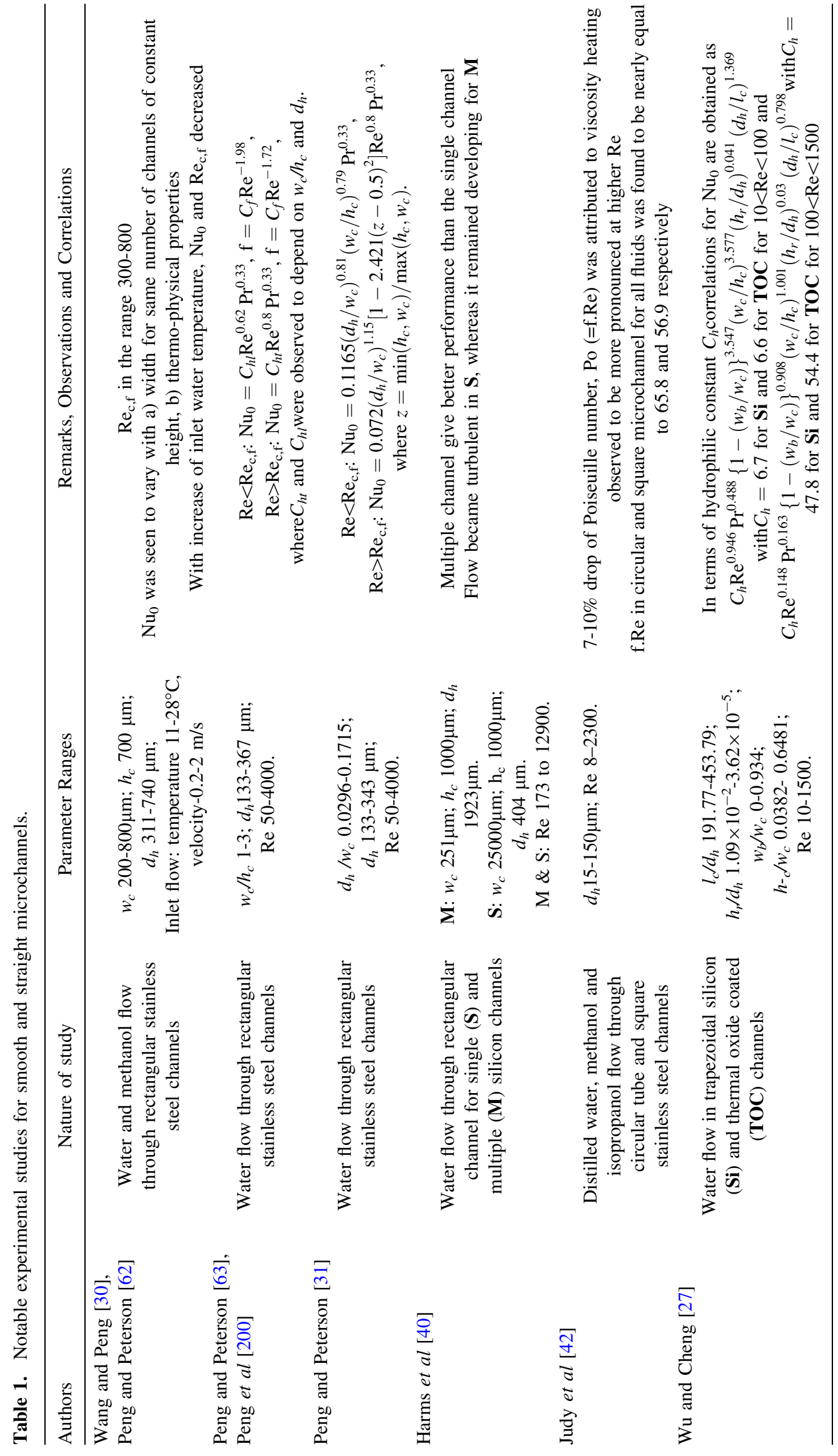


[49], Mishan et al [50] and Xu and Song [51] particularly described the entrance length effect on their numerical predictions. Numerical results of $\mathrm{Qu}$ and Mudawar [43] showed pressure drop to decrease and heat flux to increase with decrease in fluid viscosity, the effects being more apparent at lower Re.

In the studies of microtube flow, Gulhane and Mahulikar [52, 53] and Kumar and Mahulikar [54] incorporated the variation of conductivity, density and viscosity of water with temperature. With respect to constant fluid property model, their predictions showed significant effect on the predicted heat transfer and persistence of the entrance effect over longer distances from the inlet irrespective of the use of fully developed or uniform inlet conditions. Experiments of Tiselj et al [48] revealed higher and lower wall temperature relative to the flowing fluid respectively near to the inlet and outlet of a channel, which was attributed to axial heat conduction through the wall. Higher wall temperature zone persists over a longer channel length for higher Re. The effect of axial condition was found to depend on the conductivity ratio of wall to fluid, shape and aspect ratio of the channel [55-57].

Experiments of Judy et al [42] with isopropanol flow through microtube and square channel showed insignificant viscous dissipation at low value of Nahme number around 0.02 . Nonino et al [58] reported viscous dissipation effect to be important only near the channel entrance. $\mathrm{Wu}$ and Cheng [27] successfully compared their experimental results against the numerical prediction for water flow through a channel of hydraulic diameter as small as $25.9 \mu \mathrm{m}$. Conjugate heat transfer analysis of Kong and Wei [59] revealed a deviation in $\mathrm{f}$ and $\mathrm{Nu}$ against their experimental results over a range of Re from 1000 to 5500 progressively increasing up to $10 \%$ and $30 \%$, respectively beyond a high Re. Experimental studies by Liu et al [60], Jian et al [61] and Chai et al [5] with disruptive structures in an otherwise smooth channel indicated high Re flow to produce disproportionately high pressure drop over the heat transfer enhancement.

Harms et al [40] demonstrated a multichannel system to be more effective than a single-channel system for the same pumping power. The increase in overall pumping power, and hence in the size of the pump, reduces the portability of compact devices. In their experimental study with rectangular microchannel for Re in the range of 20-4000, Xu et al [41] observed a rapid increase in $\mathrm{f}$ for Re beyond 1500 . This was attributed to an early onset of turbulence, which is consistent with the experimental finding of Peng et al $[62,63]$, pointing to a lower critical Re for transition with a decrease in the hydraulic diameter. The critical Re for transition $\left(\operatorname{Re}_{\mathrm{c}, \mathrm{f}}\right)$ was found to lie in the range between 1500 and 2200 by Pfund et al [64] for rectangular channels, by Maynes and Webb [65] for circular tubes, and by Wu and Cheng [27] for trapezoidal channels. By capturing the velocity profiles through micro-PIV in a channel of $237 \mu \mathrm{m}$ hydraulic diameter over Re range of 50-2800, Hao et al 


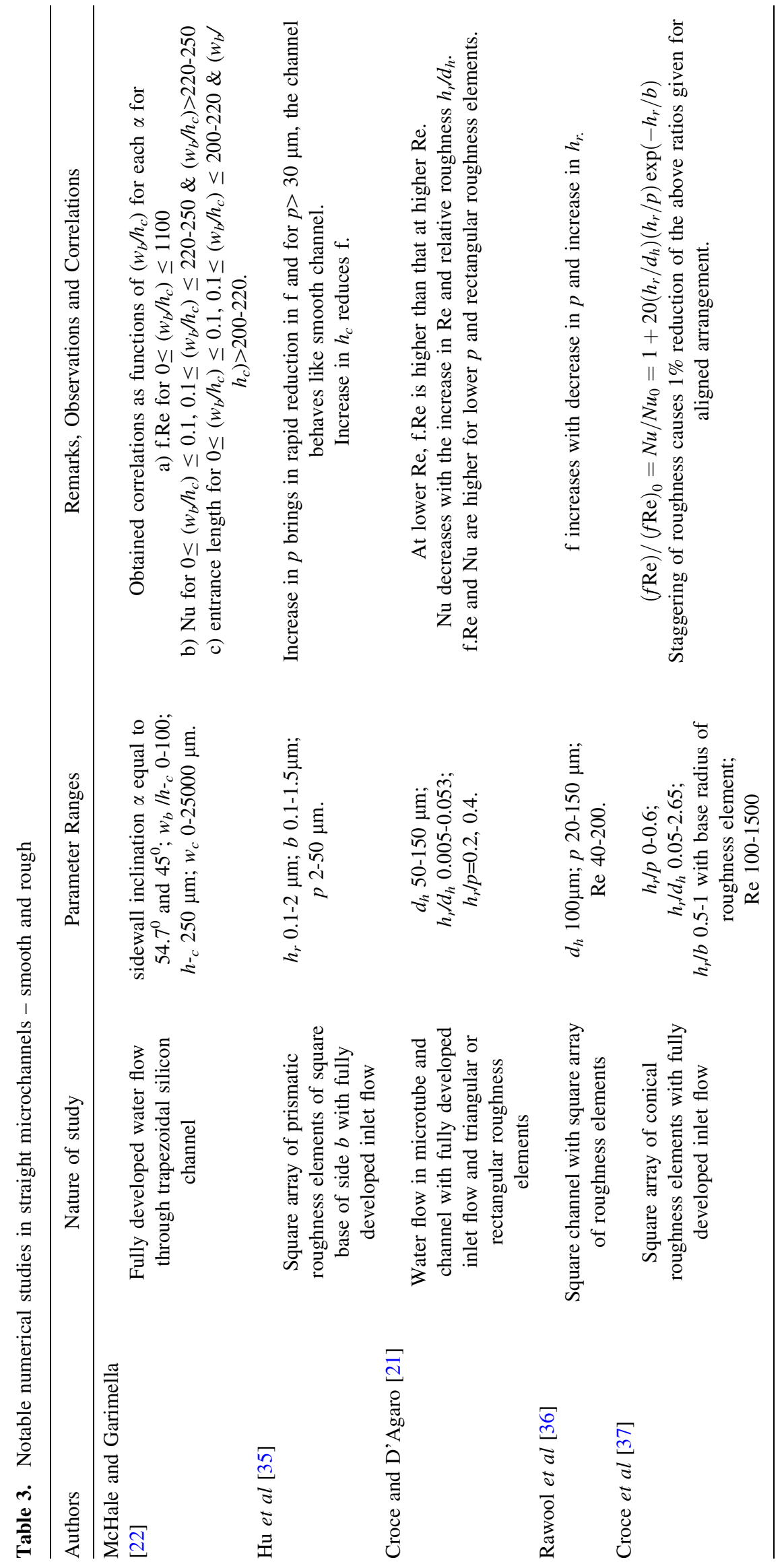


[66] showed the transition in the observed f-Re variation to be due to the entrance effect that is known to persist over a longer distance from the inlet with an increase in Re. The existence of a non-fluctuating uniform velocity distribution in the channel core is indeed an evidence of the persistence of the entrance length.

Developing thermal boundary layers cause a rise in heat transfer in microchannel in the entrance zone. Experiments of Wang and Peng [30] and Liu et al [60] exhibited entrance effect at low Re with increase in inlet flow temperature and wall roughness respectively. Liu et al [60] observed an increase of $\mathrm{f}$ above $\mathrm{Re}$ of 1100 and $\mathrm{Nu}$ above Re of 500 in their steel-tube experiments. In order to keep the pump size small together with achieving the desired heat transfer enhancement, $\mathrm{Re}$ is maintained around or below 1000 in many practical devices irrespective of whether the sharp rise in $\mathrm{f}$ with $\mathrm{Re}$ is due to an earlier onset of flow turbulence or an increased entrance length. Microchannels of smaller hydraulic diameter require high pumping power $[41,42,60]$, which may cause leakage in microsink and disrupt the electronic device. Syringe pumps or micro-pumps that are popular in micro-sink systems for their small size and weight are unlikely to provide high pumping power.

The lack of turbulence in flows with Re below 1000 requires more innovations in order to meet the demand of heat removal rate that Ghani et al [67] presently peg at close to $300 \mathrm{~W} / \mathrm{cm}^{2}$ for high-performance integrated chips. Ebadian and Lin [68] suggest that this rate will go up to even $1000 \mathrm{~W} / \mathrm{cm}^{2}$. Boyd [69], Lee and Mudawar [70], Tullius et al [71] and Majumder et al [72] regard boiling flow or two-phase liquid-gas flow as the mean for enhancing the heat transfer. Kandlikar et al [73] provided a comprehensive review in this regard. Mudawar [74] and $\mathrm{Li}$ and $\mathrm{Wu}$ [75] mentioned catalytic reactors, heat pipes, micro-evaporators for fuel cells and fuel injection systems for internal combustion equipment as the possible application areas of electronic and avionic industry. However, the requirement of deploying an additional condenser in the heat transfer loop makes boiling an unattractive proposition for electronic and optical devices not involving extremely high heat-flux like nuclear fuel rods. Agostini et al [76] reviewed and found that two phase flow boiling is a prominent technique to improve the performance of equipment for heat flux greater than $1 \mathrm{MW} / \mathrm{m}^{2}$. The pressure drop in two-phase is more than in single-phase flow and the dynamics of vapor bubble is influenced by the shape and size of the channel.

Use of special coolants like ammonia or R134a, as considered by Adham et al [10] and Lee and Mudawar [70], for cooling of electronic devices remains a challenge to implement, as observed by Kandlikar [77], Kandlikar and Grande [19] and Kenny et al [78]. Ease of maintenance and fault-tolerant operation over varied situations call for simple component profiles. Sharma et al [79] estimated the energy consumption for thermal management of microelectronic systems at big-data centers at $33 \%$ of the overall consumption. Active micro-devices involving pumping action, say by electro-osmosis of an ionized polar liquid, generating flow with $\mathrm{Re}$ of the order of 1 or lower as discussed for instance by Bayraktar and Pidugu [80] and Biswas et al [81], have been considered essentially for flow mixing with low heat transfer implications. Their target use remains confined by and large to tiny biomedical lab-onchip devices.

Tsia and Chein [82], Li and Kleinsteuer [83], Bhattacharyya et al [84], Mohammed et al [85, 86], Gunnasegaran et al [23], Ahmed et al [87], Salman et al [88], Noh et al [89], Abubakar et al [90] are among the researchers, who predicted that the increase in $\mathrm{Nu}$ gets somewhat offset by the accompanying rise in $\mathrm{f}$ with an increase in the volume fraction of nanoparticles. Such particles of metal, metallic oxide, diamond or carbon are used under dispersed condition in water, engine oil, glycerine or ethylene glycol. The rise in $\mathrm{Nu}$, typically studied in $0-5 \%$ volume fraction range, arises from a higher effective thermal conductivity of the nanofluid brought about by the high thermal conductivity of nanoparticles. However, stable, non-corrosive and long-term use of these fluids has not yet been established. In a more general context, Das et al [91] reviewed the challenges of preparing nanofluids and their potential in enhancing heat transfer by virtue of much higher thermal conductivity relative to the fluid in which the particles remain in suspension. Anoop et al [92] reported a continuous degradation of the channel surface due to precipitation of nanoparticles from the nanofluid.

A notable effect of the presence of nanoparticles in the base fluid is the alternation of both the effective thermal conductivity and viscosity for the same average flow velocity. Li et al [93] depicted this effect in terms of the variation of inlet velocity and $\mathrm{Re}$ for different volume fraction of nanoparticles. Akbarinia et al [94] summarised from their finite-volume analysis that the heat transfer enhancement is more an outcome of increased flow velocity required to keep Re comparable to the flow of only the base fluid. Toh et al [95] confirmed that the increase in fluid viscosity can have a significant impact on the overall pressure drop. A numerical study by Islami et al [96] on nanofluid flow through a rectangular microchannel with baffles placed alternately at the top and bottom walls revealed greater heat transfer augmentation due to vortices created by the baffles than the higher conductivity of nanofluid. However, the experimental study of Anbumeenakhsi and Thansekhar [97] exhibited TEF to increase beyond 3.0 with addition of $0.25 \%$ (by volume) aluminum oxide nanoparticles in water. The numerical results of Li et al [93] in the context of flow with wall disruption in the form of dimples in the side walls of microchannel revealed the increase in TEF to be due to a marginally lower rise of $f$ than that of $\mathrm{Nu}$ with increase in nanoparticle volume fraction.

Continuous development of thermal boundary layer in smooth microchannel increases the resistance to heat 
transfer. The boundary layer can be broken at multiple edges created by placing disruptive structures at small distances within the flow passage in an otherwise smooth channel. Using this idea, researchers have proposed various innovations for implementation in electronic devices as reviewed in the next section.

\section{Channels with flow disruptions}

Ahmed et al [87] and Dewan and Srivastava [98] reviewed the use of flow disruptions for achieving better flow mixing and higher heat transfer. Some popularly used structures are dimples and protrusions (figure 2) and cavities and ribs (figures 4 and 5). The hybridization of these structures and different arrangements of fins and interruptions (some of which are depicted in figure 7) and vortex generators (figure 9) can be further utilized. Of course, these structures are larger than $4 \mu \mathrm{m}$ that was considered by Zhang et al [99] for simulating surface roughness in their computational study. Even such tiny protrusions exhibited spiked disruptions of local $\mathrm{Nu}$ distribution in the vicinity of triangular, rectangular or semi-circular roughness. The spikes in the pressure variation were however not that prominent. Aligned and staggered arrangements of inclined delta and rectangular wings and winglets (termed as vortex generators or VGs) between the channel floor and roof have also been used. The underlying enhancement mechanism is periodic disruption or destabilization of the continuously growing boundary layer at the sharp edges of the structures. This is achieved either by tripping the boundary layer, by contracting the flow passage or by creating oscillating and advective vortex structure in the regions of flow expansion. With respect to these two-dimensional structures, threedimensional local protrusions and dimples result in less extensive disruptions. Use of wall jets has also been contemplated for interrupting boundary layers locally. These interruptions are especially important for Re well below 100 that are encountered in lab-on-a-chip micro-devices like chemical and biological assays. The advantage of vortex shedding is getting extensively researched in other areas too.

\subsection{Dimples and protrusions}

A number of numerical studies have been conducted with dimples and protrusions on the walls of microchannels. Figure 2 shows linear and staggered arrangements of these structures, each as a spherical surface with a footprint diameter of $d_{f}$ and depth or height $D$ on an otherwise smooth side wall of width $w_{w}$.Wei et al [100] and Xu et al [101] considered a linear array of internal dimples centered along the bottom wall of a rectangular microchannel. Wei et al [100] employed a constant heat flux of $0.1 \mathrm{~W} / \mathrm{mm}^{2}$ across the side, top and bottom walls, whereas $\mathrm{Xu}$ et al [101] considered heat flux of $1 \mathrm{~W} / \mathrm{mm}^{2}$ at the bottom wall

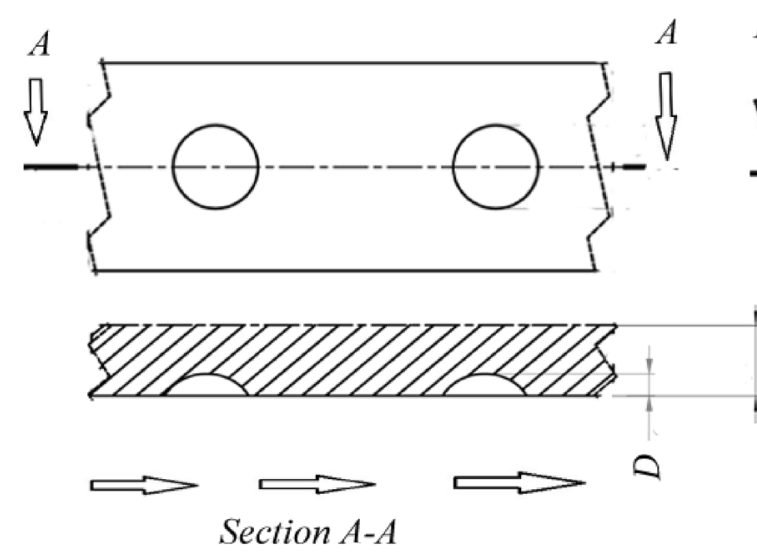

(a)

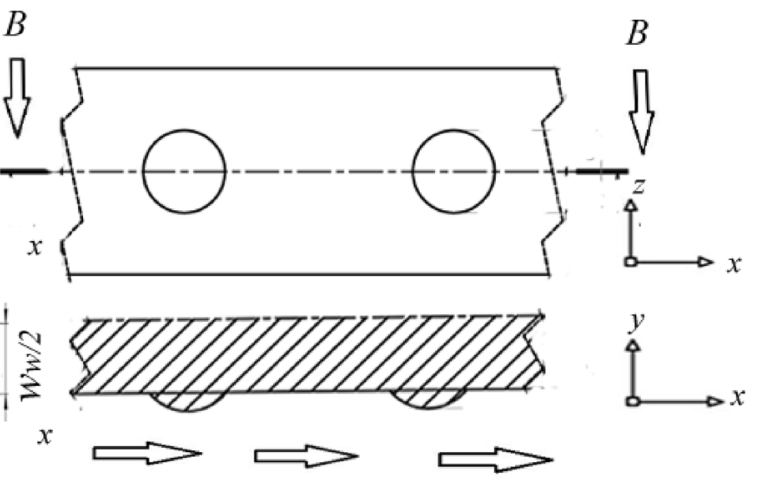

Section $B-B$

(b)

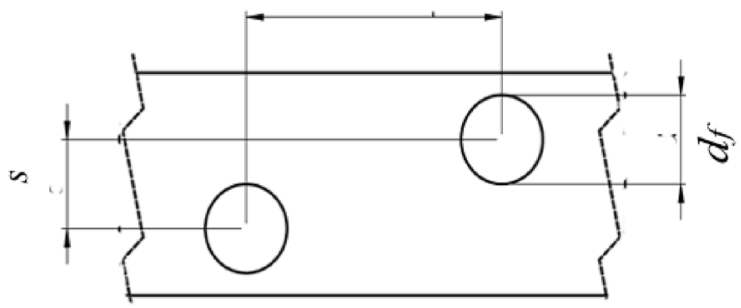

(c)

Figure 2. Dimples and protrusions on a side wall of a channel with $(a)$ aligned dimples, $(b)$ aligned protrusions, $(c)$ staggered dimples or protrusions. 
along the central $10 \mathrm{~mm}$ length of the $20 \mathrm{~mm}$ long and 0.5 $\mathrm{mm}$ wide channel with flow at Re of 500. All the studies revealed no substantial change in $\mathrm{f}$, but an increase in $\mathrm{Nu}$ with respect to a smooth channel. The heat transfer is observed to be augmented in the shear layer reattachment region downstream of a dimple while it is reduced in the low-velocity recirculation zone within the dimple [100, 102]. With respect to aligned dimples, a staggered arrangement provides higher heat transfer due to non-uniform and asymmetric structure of vortices. However, longer pitch and higher Re produce higher drag force and hence higher pressure drop.

On the basis of three-dimensional streamline plots, $\mathrm{Xu}$ et al [101] concluded the effects of increased channel aspect ratio from 1 and the dimple depth to be similar. For low value of either of the parameters, recirculation bubble remains confined in the bottom part of a dimple. With increase in any parameter value, the bubble splits into two symmetric forced vortices that gradually expand in size so as to cover the entire dimple volume. In a higher aspect ratio channel of fixed width, clearly the flow disruption effect is stronger due to higher mean flow velocity with the vortices becoming stronger and developing into cyclonic patterns, each with a distinct eye. Of course, a low-velocity zone exists near such an eye that expectedly weakens the convective circulation locally, while strengthening it away from the eye. The strengthening effect is apparent in their predicted $\mathrm{Nu}$ distribution which shows a local increase downstream of the eye beyond the dimple. This increase corresponding to each dimple in turn increases the surface averaged $\mathrm{Nu}$. An effect of higher dimple depth can be construed as a barrier to the propagation of the eye effect beyond the dimple. This explains the achievement of the highest surface-averaged $\mathrm{Nu}$ for an intermediate dimple depth. Since an increase in the aspect ratio does not introduce the barrier effect, the average $\mathrm{Nu}$ keeps on rising with an increase in the aspect ratio.

The effect of increased mean velocity studied by Lan et al [102] and $\mathrm{Li}$ et al [93, 103] exhibited limiting streamline patterns similar to that reported by $\mathrm{Xu}$ et al [101]. It was evident [102, 103] that dimple-protrusion combination on opposite side walls would result in higher TEF than dimples on only one side wall. At low Re, low heat transfer zone due to the large recirculation within a dimple gets suppressed by the flow deviation caused by a protrusion on the opposite wall. At higher Re, the presence of protrusion increases the form drag and hence the pressure drop of channel. In comparison to an aligned arrangement, a staggered pattern of dimple and protrusion with small pitch yields higher TEF, presumably due to the higher flow turning effect. Lan et al [102] considered dimples of $20 \mu \mathrm{m}$ depth and footprint diameter equal to 100 $\mu \mathrm{m}$ and in terms of $\operatorname{Re}$ and the ratio of axial pitch $p$ and footprint diameter of dimples or protrusion $d_{f}$ (Fig. 2). They obtained enhancement correlations of the form

$$
\mathrm{f} / \mathrm{f}_{0}, \mathrm{Nu} / \mathrm{Nu}_{0}=a+b \operatorname{Re}^{m}\left(p / d_{f}\right)^{n},
$$

where $a, b, m$ and $n$ are setting-dependent constants. Similar coefficients proposed by Li et al [93, 103] include two separate ratios $\left(p / d_{f}\right)$ and $\left(s / d_{f}\right)$ over and above Re, Prandtl number and volume fraction of nanoparticles. Mentioning their applicability in petrochemical, power, pharmaceutical and food industries, Li et al [103] considered water-CMC solution with CMC concentration varying in the range of 100-4000 ppm and modelled the behaviour as a power-law fluid. Li et al $[93,103]$ found significant effects on TEF of the concentrations of $\mathrm{CMC}$ or $\mathrm{Al}_{2} \mathrm{O}_{3}$ nanoparticles in water.

Li et al [104] fed the numerical predictions to a pattern search algorithm for TEF optimization with repeated arrangements of a dimple on a side wall followed by a pin fin in a rectangular microchannel. The maximum TEF of 2.11 was predicted at $\mathrm{Re}$ of 300 for $52 \mu \mathrm{m}$ pin diameter, 10 $\mu \mathrm{m}$ depth of dimple for $100 \mu \mathrm{m}$ footprint diameter, and 150 $\mu \mathrm{m}$ center-to-center distance between two consecutive pins and dimples. The comparison of the results of this optimal case with those of $\mathrm{Li}$ et al [93, 103] along with the notable aspects of the studies is summarized in table 4 and figure 3. This table and the figure bear cross referencing. Huang et al [105] considered convex, concave and mixed dimples on the bottom wall, flow inlets at the top in the form of impinging jets on the bottom wall structures and outlets at both ends of the rectangular basic channels. The highest $\mathrm{Nu}$ was obtained for convex dimples followed by channels without dimples, with mixed dimples and with concave dimples.

$\mathrm{Xu}$ et al [101] predicted only $15 \%$ increase in $\mathrm{Nu}$ and $2 \%$ drop in $\mathrm{f}$ together contributing to $16 \%$ increase in TEF. The optimal setting of the channel aspect ratio, the radius, depth and spacing of the dimples were obtained as $4,0.2 \mathrm{~mm}, 0.1$ $\mathrm{mm}$ and $0.7 \mathrm{~mm}$, respectively for the centres of the first and last dimples located $2 \mathrm{~mm}$ and $16 \mathrm{~mm}$ away from the inlet, respectively. While TEF was found to increase with increase in the channel aspect ratio and decrease in the dimple spacing, the existence of an optimum depth for the highest $\mathrm{Nu}$ was predicted. Higher rise in TEF with protrusion and dimple as depicted in table 4 and figure 3 suggests that protrusion may be a good disruptive structure, in which the strong heat transfer enhancement effect easily compensates for the marginal rise in pressure drop penalty. The structures like cavities, ribs and fins extend through the entire channel depth or nearly so. Hence, stronger flow disruption effects are expected from such structures, either individually or in combination.

\subsection{Cavities and ribs}

Different arrangements of cavities, or grooves, and ribs mostly on the side walls of rectangular and triangular microchannels with some intermediate gaps between two consecutive structures has been investigated numerically, 


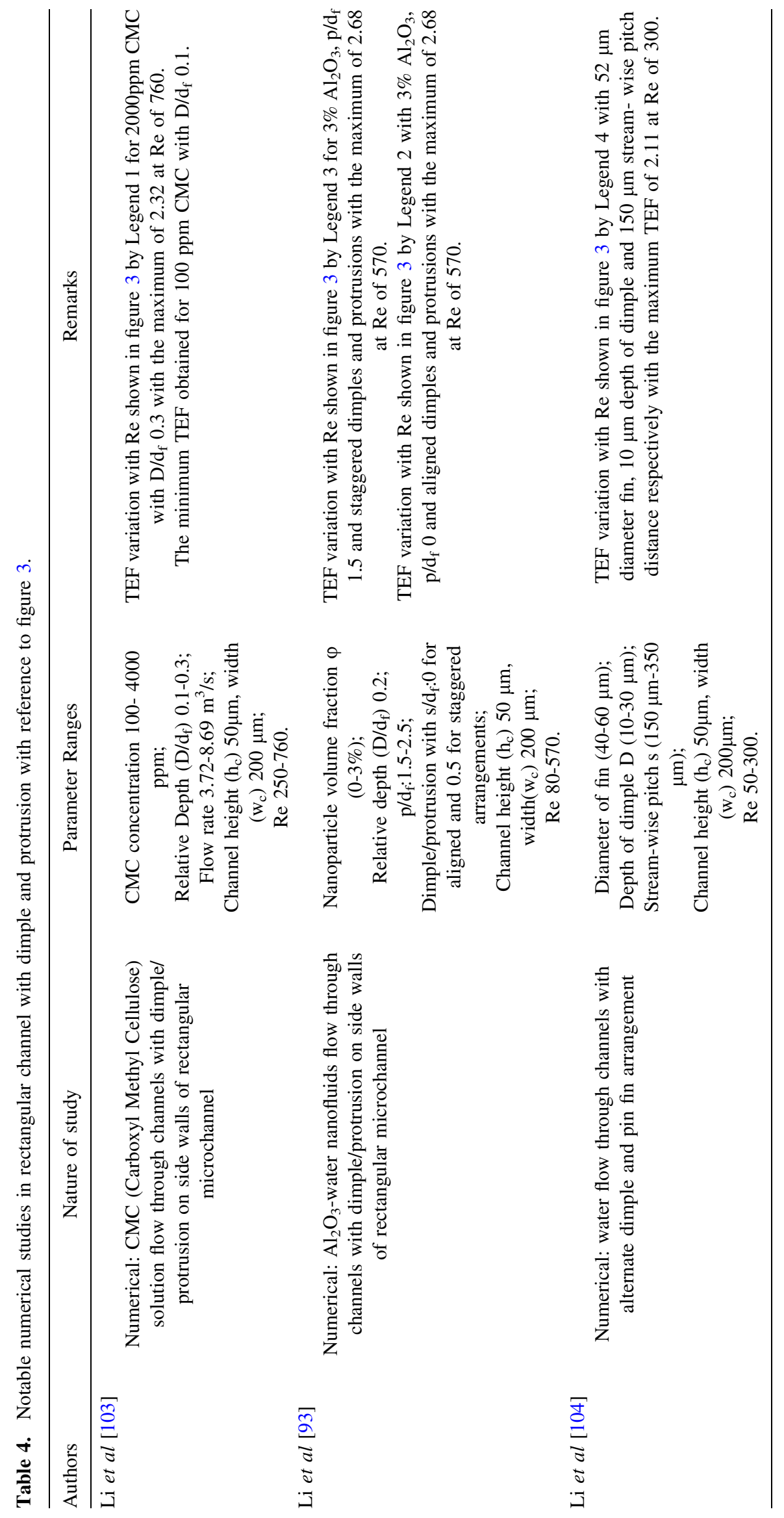




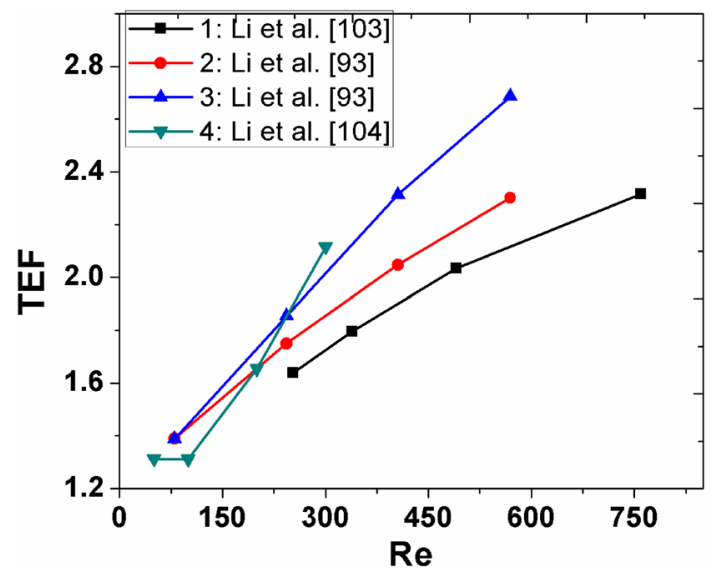

Figure 3. Optimum TEF predictions for channels with dimples and protrusions with legends explained in the last column of table 4.

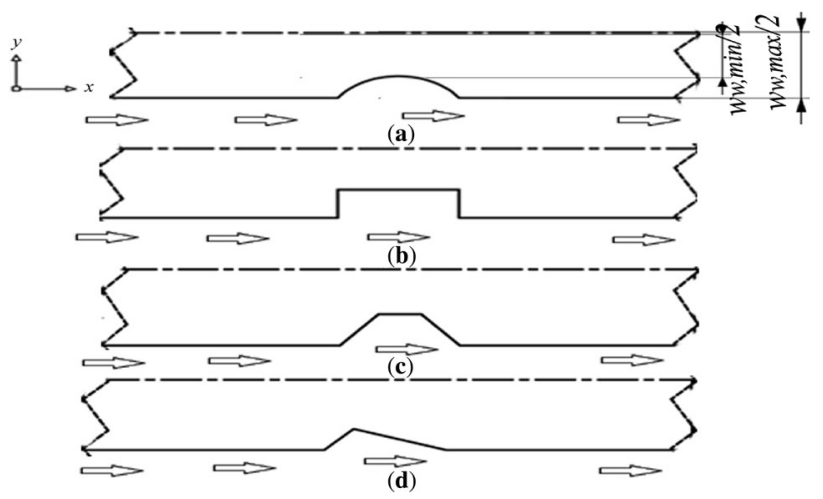

Figure 4. Half-width of an intermediate side wall for rectangular microchannels with $(a)$ circular or fan-shaped cavity, $(b)$ rectangular cavity, $(c)$ trapezoidal cavity and $(d)$ triangular cavity.

mostly as a laminar-flow conjugate heat transfer problem. Al-Asadi et al [106] considered semi-cylindrical or quartercylindrical ribs with transverse axis on the bottom wall. Their analysis revealed no improvement in TEF beyond 1.05 , irrespective of whether the ribs were extended along the entire channel width or had gaps either at both sides or two sides as well as in the middle. Some popular modifications of the side walls are triangular, trapezoidal and arc forms are shown in figures 4 and 5. While arc form structures are often mentioned as fan-shaped, the cavities are referred also as re-entrant cavities. It may be incidentally mentioned here that Xia et al [107] performed a conjugate heat transfer analysis of the entire system with rectangular channels shown in the left of Part (a) of figure 1 together with Parts (b) and (c) depicting different headers and inletoutlet connections, respectively. It was found that rectangular header with I-type connections provides performance better than the other arrangements by ensuring a better flow distribution through the channels. A comparison with smooth walled rectangular channel against those with

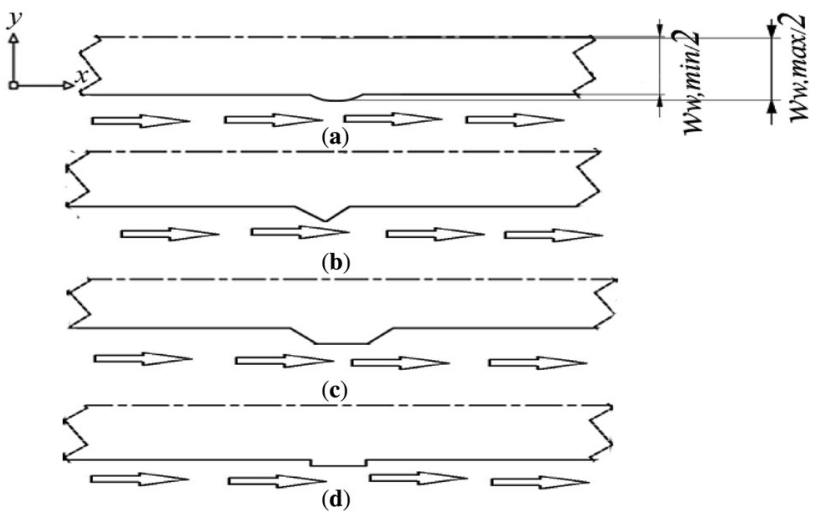

Figure 5. Half-width of an intermediate side wall for rectangular microchannels with (a) circular rib, (b) triangular rib, (c) trapezoidal rib and $(d)$ rectangular rib.

triangular and fan-shaped cavities clearly exhibited the enhancement potential of cavities.

For fan and triangular cavities in the Re range of 150-600, Xia et al $[108,109]$ obtained the optimum ratio of the length between two nearest aligned fan-shaped and triangular cavities to the length of a cavity, and the maximum to minimum channel widths was found to be in the range of 0.15-2 and 1.4-2.1, respectively. Chai et al [110] found that the effect of offset in the structure on the facing walls is not significant. Kuppusamy et al [111, 112] predicted better performance of triangular grooves over trapezoidal ones along with performance improvement with increase in both angle and depth for triangular grooves. With respect to smooth channels, Liu et al [45] predicted about $60 \%$ rise in $\mathrm{Nu}$ with triangular grooves in the channel bottom surpassing the gain achievable from other structural forms considered in their study. Lower drop in local $\mathrm{Nu}$ along the channel length was noted as an advantage of the grooved channels. The edges of a cavity cause discrete disruption of the thermal boundary layer. Especially at higher Re, higher flow mixing is also expected due to the diverging and converging flows near the cavity entrance and exit, respectively. These mechanisms contribute towards augmentation of heat transfer. However at lower $\mathrm{Re}$, the formation of recirculation bubble widest near the central edge of a triangular cavity impedes the heat transfer, admittedly accompanied with a drop in pressure due to the decreasing shear effect of the main flow slipping under a bubble. Ahmed and Ahmed [113] expectedly observed the widest recirculation zone in a rectangular cavity having large angles between the faces and the reduction in area, dead zone at the tip of triangular cavity reduce the heat transfer. In comparison to a triangular cavity, the absence of the central edge in a trapezoidal channel along with its accompanying recirculation bubble makes the heat transfer effect stronger. As regard to TEF with cavities on the opposite walls, their investigation revealed symmetric arrangement to be better than asymmetric one with offset. 
Experimental investigation by Chai et al [5] in an arrangement of rectangular headers with central common line showed: (1) the corresponding numerical predictions by Xia et al [108, 109] for $\mathrm{Nu}$ and $\mathrm{f}$ to be marginally lower, (2) the parameters for triangular cavities to be slightly higher than those for fan cavities, and (3) $\mathrm{Nu}$ enhancement of about $80 \%$ with respect to that in straight channels at corresponding Re. A remarkable finding of these studies with cavities in channels is the steeper trend of monotonic variation of $\mathrm{f}$ with $\mathrm{Re}$ crossing the corresponding variation for smooth channels at around $\mathrm{Re}$ of 300 . Over the Re range of 100-700, Ahmed et al [114] predicted an increase in both $\mathrm{Nu}$ and $\mathrm{f}$ with increase in the depth of trapezoidal groove, but decrease in $\mathrm{Nu}$ and increase in $\mathrm{f}$ with increase in groove length. Kuppusamy et al [111, 112] considered flow of water, glycol or engine oil mixed with nanoparticles of $\mathrm{Al}_{2} \mathrm{O}_{3}, \mathrm{CuO}, \mathrm{SiO}_{2}$ or $\mathrm{ZnO}$ of $25-80 \mathrm{~nm}$ size, whereas Ahmed et al [114] considered copper-water nanofluid. The numerical results of Ahmed et al [114] exhibited a rise in both $\mathrm{Nu}$ and $\mathrm{f}$ with increase in particle volume fraction, while Kuppusamy et al [111, 112] reported performance improvement for smaller particle size.

The application of rib is another useful approach due to the redevelopment of boundary layer and by bringing chaotic convection in the channel. A numerical study by Xia et al [115] and Zhai et al [116] exhibited enhancement in $\mathrm{Nu}$ with respect to those due to Xia et al [108, 109] and Chai et al [110] arising from inclusion of circular ribs between two successive fan cavities especially for larger relative rib height, defined as the ratio of rib height to hydraulic diameter. The cavity and rib contribute towards negative pressure and positive pressure, respectively. The combined effect can cause acceleration, recirculation, separation and reattachment of fluid in microchannel resulting in augmentation of TEF with rise in heat transfer and pressure drop. The heat transfer is more at the downstream of rib and less at the upstream part of the cavity. With respect to a smooth channel, $\mathrm{Nu}$ and $\mathrm{f}$ have been predicted to rise by 1.3 to 3 times and up to 6.5 times.

A numerical study by Bi et al [117] in the context of mini-channels clearly revealed falling trends of $\mathrm{Nu} / \mathrm{Nu}_{0}$ and TEF with increase in $\mathrm{Re}$ in the range of 2700 to 6100 for all the cases of side walls involving either fan-shaped grooves or low-height rectangular ribs or dimples. On the lower and higher sides of Re of about 3500, TEF of dimpled channel was found to be worse and better than the grooved channel across the TEF value of about 1.25. This may be attributed to nearly constant and increasing variations of $f / f_{0}$ with $R e$ respectively for dimpled and grooved walls. The TEF was found to be the lowest in case of the ribbed channels falling even lower than unity for Re beyond 4700 .

Chai et al $[118,119]$ predicted a potential benefit of fanshaped ribs as so to counteract the drop in local $\mathrm{Nu}$ along the channel axis. Based on entropy generation analysis for rectangular, triangular, trapezoidal and fan-shaped ribs placed between fan-shaped cavities, Zhai et al [116] predicted superiority of trapezoidal and fan-shaped ribs respectively below and above Re of 300. Zhai et al [120] with triangular and fan-shaped cavities exhibited better performance for triangular cavity with trapezoidal rib for $\mathrm{Re}$ in the range of 100-300 and with triangular rib for $300<$ $\operatorname{Re}<600$. Ghani et al [121] considered microchannels with rectangular rib (MC-RR), secondary branches (MC-SOC), and both (MC-SOCRR). The highest TEF of 1.98 was obtained for MC-SOCRR through optimization of the geometrical parameter. An important observation for secondary channel is the significant contribution to the reduction of pressure drop induced by the ribs.

Xia et al [122] achieved highest TEF of 1.24 at the highest $\mathrm{Re}$ of 611 in their experiment in a microchannel with fan-shaped cavity and rectangular headers each with a common line at the mid-span of the header. The vital role of cavities and ribs in improving the TEF of microchannel is evident from figure 6. The geometrical parameters of cavity only, cavity combined with rib in side wall, and cavity in combination with centrally placed rib are shown in table 5. It is clear that the cavities on the opposite side walls without additional features do not cause a significant increase of TEF. The highest TEF is predicted with central ribs, whereas the other case of cavities and ribs on the facing side walls provide TEF in the intermediate range.

Li et al [123] predicted the best TEF of 1.619 for the combination of triangular cavity with rectangular rib on opposite walls. Datta et al [124] and Li et al [123] considered ribs on the bottom wall instead of side wall, with the ribs in the form of backward triangular, rectangular, diamond and forward triangular rib within trapezoidal cavity. They also considered rectangular rib within the triangular cavity placed centrally across the height of a rectangular channel. The contribution of rib is to reduce the size of the recirculation zone through increase in velocity of fluid within the cavity, thereby enhancing the heat transfer significantly. With a diamond rib in a trapezoidal cavity,

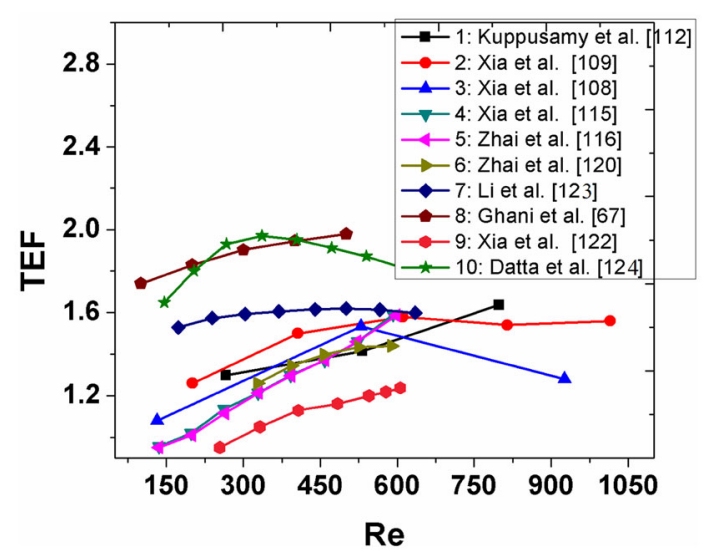

Figure 6. Optimal TEF predictions for channels with cavities and ribs with water as base fluid for with legends explained in last column of table 5 . 


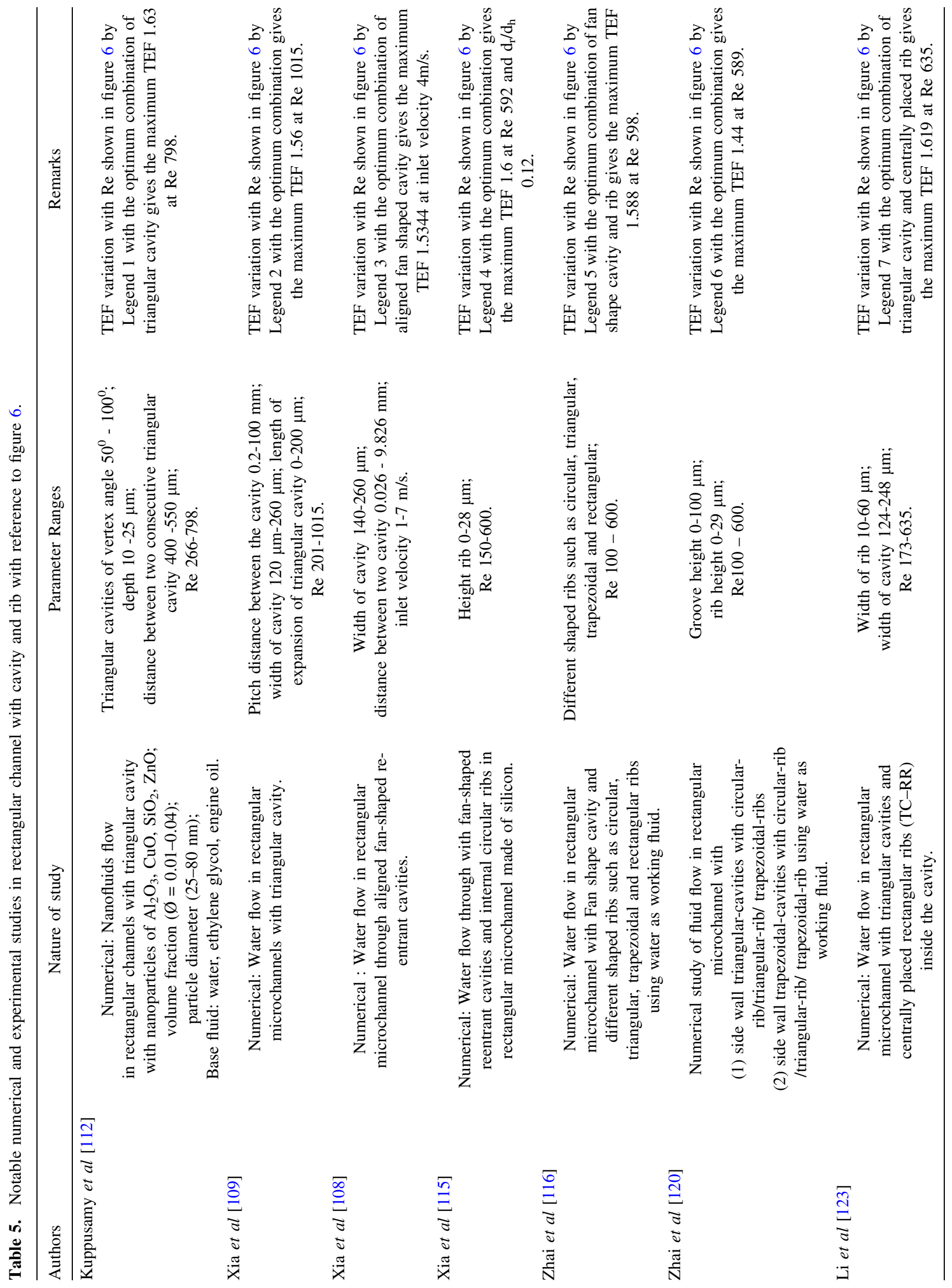


Datta et al [124] predicted the highest TEF equal to 1.97 and 2.31 for channels made of silicon and copper, respectively. Chaotic convection and lower drag force offered by the diamond rib cause a significant improvement in the performance.

Several studies revealed that the use of cavities in side walls have the potential to increase the heat transfer by disruption of thermal boundary layer at regular gaps. The diverging and converging flows respectively at the entrance and exit of a cavity lead to a rise in heat transfer, while the recirculation zone within the cavity leads to a decrease in heat transfer and pressure drop. So, ribs and cavities on the facing side walls could increase the velocity in the core zone of microchannel, but they fail to remove the recirculation zone in cavities. Among all the researches, the arrangement of central ribs on the bottom wall of a microchannel facing two cavities on the opposite side walls seems to be the most promising. Secondary channels on the side connecting the longitudinal channels near the ribs could mitigate the flow blocking effect of the ribs and the corresponding performance enhancement calls for assessment in the near future.

\subsection{Fins and interruptions}

Fins and interruptions can be interpreted as deep ribs and cavities, respectively. Figure 7 depicts some popular arrangements. Of course, the higher area of the bounding wall brought in by the higher depth is responsible for higher $\mathrm{Nu}$ and $\mathrm{f}$. In comparison to the ribs, fins are generally longer in lateral or longitudinal direction. On the other hand, interrupts are cavities deep enough to interconnect the parallel longitudinal channels. Oblique interconnections are also referred to as secondary channels. A series of interconnections often having a common lateral axis forms what is called a transverse channel or a microchamber. In the interrupted arrangement, the number of transverse channels is fewer than that of longitudinal channels. Strip fins shown in figures 7 (c) and (d) can be envisaged as sets of multiply broken walls with axes parallel to the side walls. In simple offset arrangement, two alternate sets of linear array of broken walls have an axial offset between the leading edges of the corresponding walls in two adjacent arrays. This arrangement disrupts the flow several times after every short length of the flow between two parallel fins by impacting on the leading edge of another fin belonging to the alternate set, thereby enabling the bifurcating flow to attain a more uniform lateral distribution. An oblique fin arrangement is provided by offset strip fins providing slanted passages in place of the lateral ones.

Foong et al [125] considered four internal fins projected from the side and top walls with the fins extended along the entire length of a square microchannel, as shown in figure 7 (a). The ratio of the projected fin length to the channel width considered in the numerical analysis was in the range 


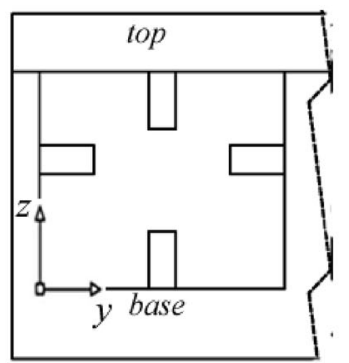

(a)

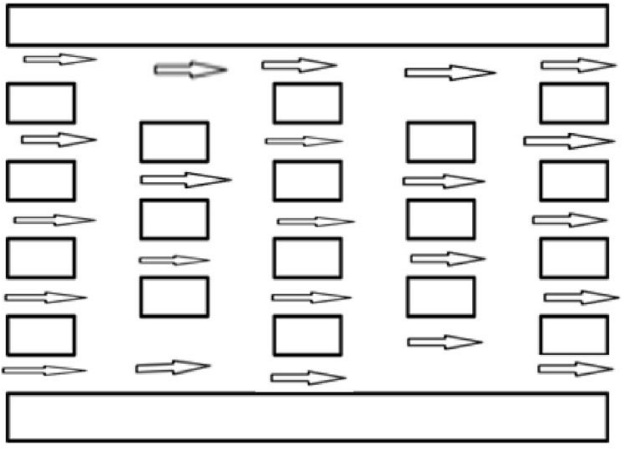

(c)

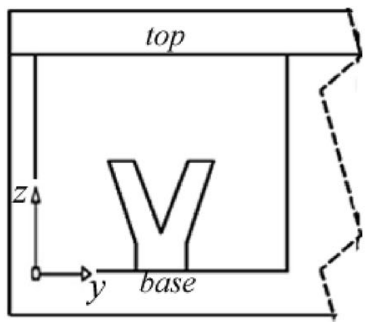

(b)

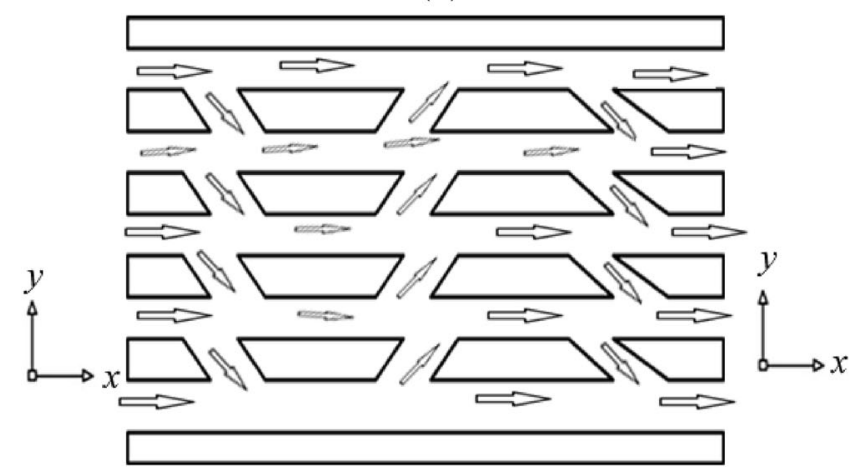

(d)

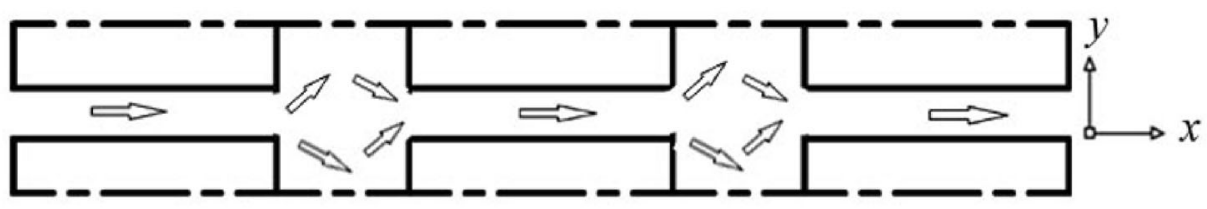

(e)

Figure 7. Rectangular microchannels with $(a)$ rectangular fins, $(b) \mathrm{Y}$ fin, $(c)$ strip fins with transverse channels, $(d)$ strip fins with oblique or secondary channels and $(e)$ microchambers.

of 0 to 0.425 . Of course, low non-zero values of the ratio resemble ribs and the zero value a smooth channel. Hydrodynamically developed but thermally developing flow was considered. A validation was provided with the smooth-channel predictions of $\mathrm{Nu}$ given by Lee and Garimella [49], who in turn validated their results against available experimental data and obtained correlations with forms quite similar to conventional ones. With increase in the projection ratio, Foong et al [125] observed an increasing trend of $\mathrm{f}$ along with a rise in $\mathrm{Nu}$ up to an optimum ratio of about 0.33 followed by a drooping trend. On the higher and lower sides of the optimum ratio, the domain extents along the channel diagonal with axial velocity below the average was observed to get elongated either towards the core or towards the corners, respectively. Near the optimum ratio, the relatively wider region with velocity higher than the average contributed to higher $\mathrm{Nu}$ due to enhanced convective heat transfer. In this arrangement, the major heat transfer enhancement is achieved from the increased conduction through the fin surfaces along with some mixing near the fin tips due to the vortex rolling up there.

Xie et al [126] considered only one longitudinal Y fin of rectangular section extended up to the channel end. This arrangement is shown in figure 7(b). Apart from the shape and number of fins, one major difference of this fin arrangement with respect to that of Foong et al [125] is the location of its leading edge at different distances away from the inlet. Since the local $\mathrm{Nu}$ keeps on decreasing in the downstream direction due to the boundary layer growth in a smooth channel, this arrangement can be considered as a ploy to augment the local $\mathrm{Nu}$ by causing a flow disruption in the form of a bifurcation at the leading edge together with providing additional heat conducting surface. The best performance was predicted by the longest fin indeed due to the larger fin area. An interesting element of this study could have been the position of the leading edge for which the enhancement in heat transfer due to the leading edge flow bifurcation would have been more than the reduction arising from not having the area contribution of the fin up to the leading edge. A study on the effect of the included angle between the two top arms of the $\mathrm{Y}$ fin revealed the best TEF as the $\mathrm{Y}$ fin assumes a $\mathrm{T}$ shape in the limit. This could be attributed to the mixing due to the rolling vortices on large unshared fluid volumes as a result of large separation between the fin tips. The nearness of the fin tips for the configuration studied by Foong et al [125] is not expected to work on a common fluid volume resulting in a weakening effect as the fin tips get closer to each other. 
Xie et al [127] analyzed symmetric vertical arrangements of one fin forming one stage, three fins arranged over two interfaced axial stages and six fins over three interfaced axial stages with all the arrangements extended up to the channel end. The leading edge of the leading fin located at the mid plane was considered at different distances away from the inlet. Later, fins were placed at uniform lateral spacing with the number of fins increasing by one through each subsequent stage. This stage-by-stage stepped increase of the conducting fin surface area ensured corresponding stepped rise of the local $\mathrm{Nu}$. While the leading edges of the fins causes enhanced mixing due to flow bifurcation, the trailing edges perhaps invigorates mixing by the action of the longitudinally shed vortices. Of course, better mixing brings in more uniformity in the local $\mathrm{Nu}$ variation, admittedly against a higher penalty of pressure drop. All these observations are supported by the results of their study. Shen et al [199] considered vertical bifurcation plate placed at the middle of channel and noticed an effective decrease of the channel temperature. Their optimization study indicated the maximum TEF to be limited to 1.145. Jia et al [128] considered cone shaped micro pin fins placed on the bottom wall of rectangular microchannels only between the inlet and the middle, or between the middle and the outlet, or concentrated about the mid-length, or equally distributed from the inlet to the outlet. A parametric study indicated the highest TEF of 1.55 for the distributed arrangement.

Both the uniformity and the penalty are expected to increase due to many more interruptions in the offset strip fin arrangements in microchannels with lateral interconnections. Colgan et al [129] demonstrated a high heat transfer capability of more than $300 \mathrm{~W} / \mathrm{cm}^{2}$ in a practical implementation of such an arrangement. Hong and Cheng [130] carried out a numerical analysis for a specified temperature of water at the channel inlet and heat flux at the bottom wall with the objective of containing the maximum wall temperature achieved at the bottom of the channel outlet at a limit. They analyzed the effects of two parameters, namely the number ranging from 1 to 20 for breaks in a wall array and the ratio of $0.5,1$ and 2 for the wall length to the gap length in the same array. Their study revealed some interesting effects.

The decrease of mass flow rate with increase in the number indicating saturation to values increasing with the ratio can be explained by the growth and saturation of the convective effect due to both the leading edge bifurcation and the trailing vortex shedding outperforming the increased wall conduction effect arising from larger fin area and thinner boundary layer. In fact, the highest pressure drop in case of the lowest ratio confirms the convective effects getting stronger with increase in the gap between the fins in the same array. The increase of this effect countered by the decrease in the mass flow rate increasing with increase in the number of gaps resulted in an initially massflow rate dominated falling trend followed by the convection-dominated rising trend of the pressure drop. As a result, the lowest pumping power requirement was predicted for the two higher ratios studied depending on the target bottom wall temperature at the channel exit. Most of these analyses pertaining to finned microchannels were mainly aimed at developing physical understanding of various enhancement mechanisms. Particularly, the studies of the strip fin arrangements lack performance comparison with respect to the corresponding smooth microchannels.

Lee et al [131] and Kuppusamy et al [132] examined heat transfer enhancement in microchannels with oblique fins, as depicted in figure 7(d). Lee et al [131] carried out a combined numerical and experimental work with slanted secondary channels all parallel to each other presenting a successful validation of their numerical prediction in terms of variations of $\mathrm{Nu}$ and $\mathrm{f}$ with $\mathrm{Re}$ for both smooth and finned microchannels. The secondary channels were identified as means of disrupting the boundary layers thereby reducing their resistance to heat transfer to the wall and enhancing mixing by the flow at the exits of the secondary channels arising from the pressure difference across the two ends due to slanting of a channel. Kuppusamy et al [132] numerically analyzed the effects of two alternate sets of parallel channels having opposite slants with a prior validation against the local variation of $\mathrm{Nu}$ along the length of a smooth channel provided by Kandlikar et al [133]. While Lee et al [131] achieved a maximum TEF of about 1.7, the numerical results of Kuppusamy et al [132] at a fixed mass flow rate indicated a maximum TEF of nearly 1.5. The alternating slants conceived by Kuppusamy et al [132] for the secondary channels is expected to provide more uniformity to local $\mathrm{Nu}$ by having mixing due to the secondary exit flow in all the channels rather than at alternate parallel channels.

Xu et al [51, 134] and Chai et al [135] considered longitudinal microchannels of triangular and rectangular cross sections with transverse interrupts respectively of trapezoidal and rectangular channel forms. Due to repeated redeveloping boundary layer, $\mathrm{Nu}$ has been predicted to be the maximum for interrupted microchannel heat sink in comparison to conventional microchannel. The sudden expansion provides pressure recovery in the interrupted zone which decreases the flow resistance with respect to conventional channels. The decrease in velocity within a microchamber creates hot spot by the rise of temperature in the micro chamber. Chai et al $[135,136]$ involved additional longitudinal ribs of rectangular section facing the longitudinal channel and located at the axial mid span of each of the wide transverse channel. The effects of rectangular, backward triangular, diamond, forward triangular, ellipsoidal ribs in the transverse micro chambers were investigated. These structures remove the hot spot in the microchamber by accelerating the fluid incurring a higher penalty in pressure drop within the microchamber. A successful experimental validation for $\mathrm{Nu}$ and $\mathrm{f}$ was accomplished in these studies for the corresponding 
configurations. The parametric optimization study of Chai et al [135] based on the numerical results exhibited a maximum TEF of about 1.5 at around Re of 320 due to the boundary layer interruptions. Additional enhancement was brought in by flow mixing in the main channel due to the interaction with the secondary channel flow sustained by the pressure difference across the slanted ends. This novelty should be appreciated against the much more complex oscillating synthetic jet configuration numerically studied by Chandratilleke et al [137], Lee at al [138, 139], Fang and Khan [140] and Kandlikar and Bapat [141].

By pulsing a diaphram, say by piezoelectric actuation, on a fluid chamber such synthetic jets could be actuated between the common chamber through orifices located on the top or bottom of each channel. While the study by Fang and Khan [140] revealed $\mathrm{Nu}$ enhancement up to 1.3 times without any appreciable change in $\mathrm{f}$, Chandratilleke et al [137] reported up to 4.1 times rise in $\mathrm{Nu}$ without citing the corresponding pressure drop. The numerical results of $\mathrm{Xu}$ et al [51] revealed substantial boosting of local $\mathrm{Nu}$ at the inlet of each interrupt along with the consequent increase to $\mathrm{Nu}$ over a wide parameter range. These local enhancements were identified as due to the elimination of the boundary layer at each inlet of the transverse interrupt. No appreciable increase in $\mathrm{f}$ below $\mathrm{Re}$ of 1000 was predicted, resulting in a significant rise in TEF. The use of microchamber decreases the pressure drop and use of rib causes increase in the velocity in the stagnant zone. The combination of microchamber and rib improves TEF to a certain extent as shown in figure 8. Among the different rib structures, the ellipsoidal ribs in microchamber used by Chai et al [136] showed the highest TEF. Important observations with variation of geometrical parameters are listed in table 6. It may be surmised that for the absence of sharp edges, ellipsoidal ribs in the micro chamber showed potentially higher TEF than ribs with sharp edges. Thus, acceleration effect of the ribs rendered by their blocking effect within microchambers seems to dominate over the boundary layer disruption effects at the sharp edges. This

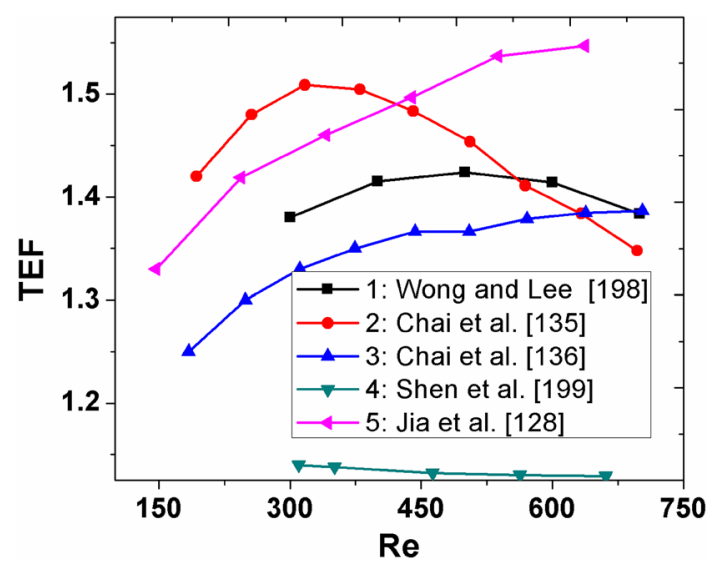

Figure 8. Optimal TEF for channels with fins and interruptions with legends explained in the last column of table 6 . construction appears to be a definite candidate for structural optimization for attaining the highest TEF.

\subsection{Vortex generators}

Another promising trend of flow disruption adapted mostly for rectangular microchannels is vortex generators, or VGs. Numerical study by Meis et al [142] and Koz et al [143] with one cylinder of circular, elliptic, square and triangular cross section placed along the height demonstrated the downstream vortices to be quite effective in enhancing both the local and average Nu. Besides heat sink and electronic chip cooling applications of vortex generators, Ahmed et al [87] provided a much broader review that included heat exchangers with flat and tubular fins and refrigeration. Vortex generator structures like wings, winglets, circular and oval fins of different orientation relative to the flow were visited. Longitudinal vortex generators, or LVGs, were seen to be more effective than transverse ones for imparting heat transfer enhancement.

Majority of the studies concerning microchannel heat sink use considered rectangular flat plates as LVGs placed at different inclinations. Figure 9 shows part of a rectangular channel with staggered arrangement of rectangular LVGs, each at an inclination of $\alpha$ with respect to the mean flow direction. Earlier studies on LVGs pertain mostly to mini channels. Such an experimental study due to Wang et al [144] revealed better performance with LVGs placed on both sides of the plane of symmetry than along only one side producing 10 to $45 \%$ enhancement in $\mathrm{Nu}$. Similar experiments of Jian et al [61] showed almost two-fold increase in $\mathrm{Nu}$ with additional penalty in $\mathrm{f}$ of only $11 \%$ in laminar flow regime contrasted by corresponding figures of $87 \%$ and $200 \%$ in turbulent regime. In a combined numerical and experimental work, Wu and Tao [145] found the best performance for VGs of $45^{\circ}$ angle placed in an air stream during the experimental study on an aluminium plate at the mid-height of a relatively bigger rectangular channel $162 \mathrm{~mm}$ wide and $62 \mathrm{~mm}$ high. The effect of VG thickness was found to be insignificant. Hsiao et al [146] predicted the beneficial effects of LVGs on fluid mixing as well.

Liu et al [147] conducted experiments in rectangular microchannels over $\mathrm{Re}$ range of 170-1200 with aligned LVGs in three or five consecutive pairs at $30^{\circ}, 45^{\circ}, 135^{\circ}$ or $150^{\circ}$ or three-pair combinations of $150^{\circ}-30^{\circ}-150^{\circ}, 30^{\circ}$ $150^{\circ}-30^{\circ}, 135^{\circ}-45^{\circ}-135^{\circ}$ and $45^{\circ}-135^{\circ}-45^{\circ}$. Chen et al [148] further considered LVG arrangements with height ratios $0.0,0.25,0.75$ and 1.0 of $\mathrm{LVG}$ height to channel height together with aspect ratios of 0.25 and 0.667 . Liu et al [147] found the combination $150^{\circ}-30^{\circ}-150^{\circ}$ to produce the best performance. They found that the flow becomes turbulent at early Reynolds number with the increase in angle and number of vortex generators. In comparison to either of the cases with height ratio of 0.25 and 1.0 , Chen 
Page 19 of $32 \quad 234$

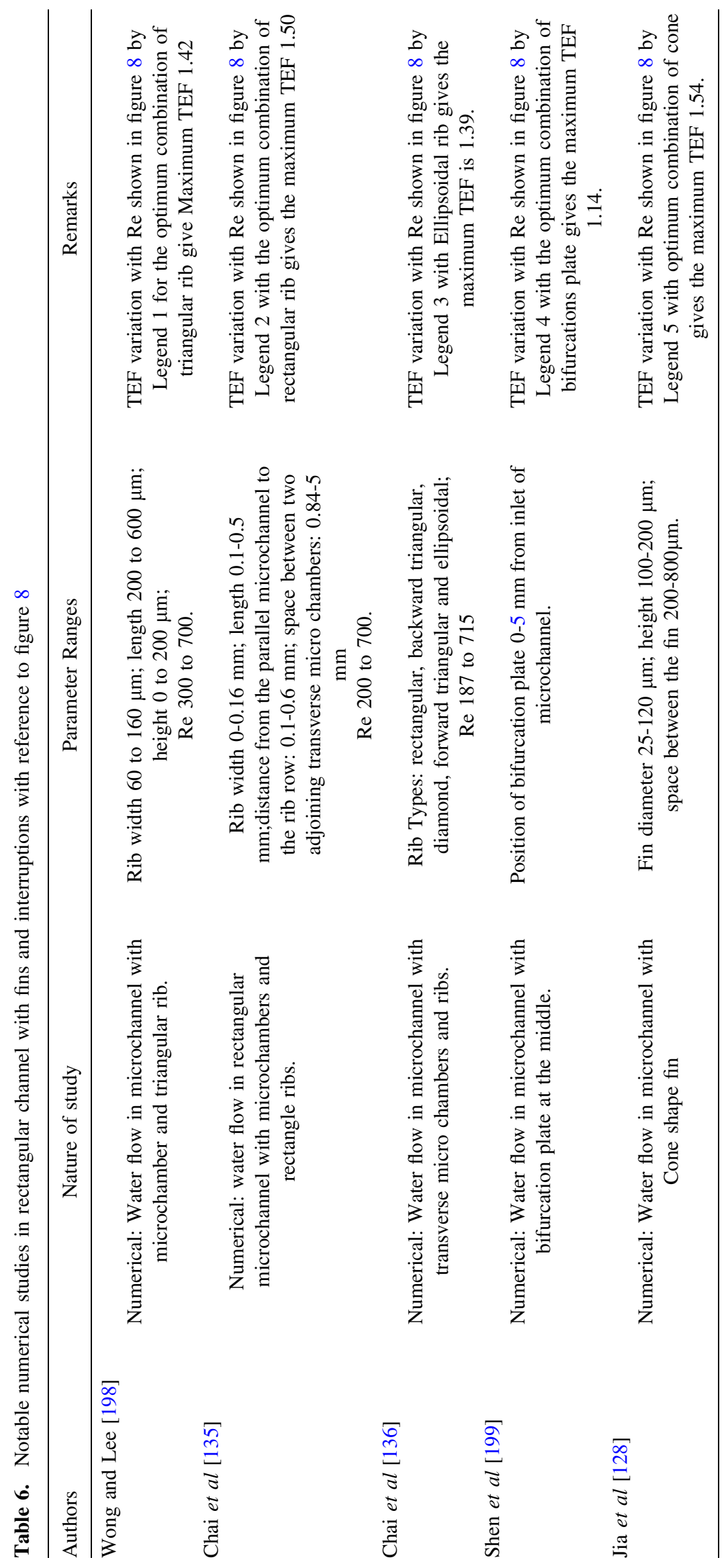




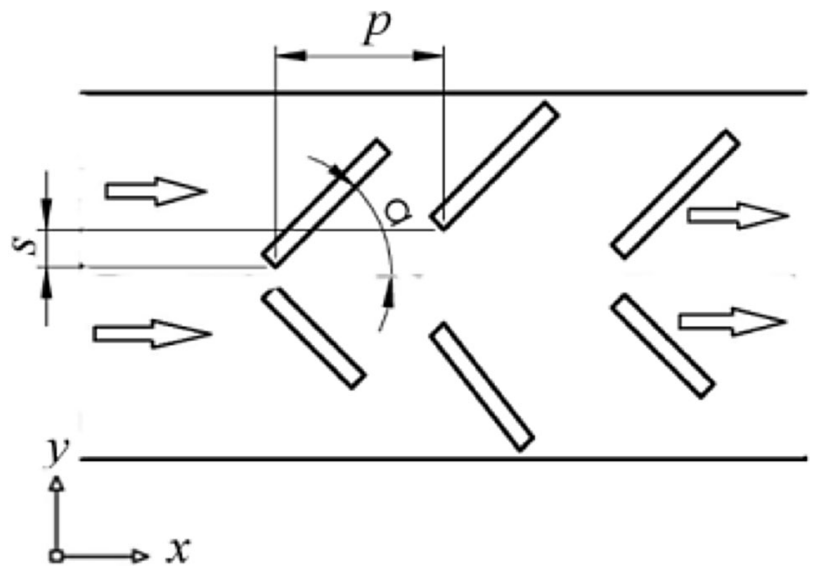

Figure 9. Portion of one channel with longitudinal vortex generators in staggered arrangement.

et al [148] observed the case with height ratio of 0.75 to yield $\mathrm{Nu}$ lower for Re less than 650 , but becoming progressively higher for Re beyond 650 . Between the values of aspect ratio equal to 0.25 and 0.0667 , their study indicated higher $\mathrm{Nu}$ to arise in the former case for LVG height ratios of 0 and 0.25 , but in the latter case for the height ratio of 1.0. However, significantly large $f$ obtained with LVG height ratio of 0.75 is a hindrance to its deployment in actual applications. These studies however do not provide the comparisons in terms of TEF. Liu et al [147] and Chen et al [148] noted longitudinal and transverse vortices arising from VGs. At low Re, VGs extending through the entire channel height generates dominantly transverse vortices rendering it ineffective for heat transfer enhancement. These vortices fail to augment the heat transfer because of short space between the VG and side wall. On the other hand, VGs occupying a part of the channel height generate longitudinal vortices that travel long distance, thereby contributing to a rise in heat transfer through improved flow mixing. But, at higher Re, the collision of the longitudinal vortices with wall deteriorates the augmentation of heat transfer. Higher width of the channels, than height, seems to be more effective for augmenting heat transfer in the channel with vortex generators.

Recent laminar-flow analyses by Ebrahimi et al [149] and Datta et al [150] exhibited excellent matching for both $\mathrm{Nu}$ and $\mathrm{f}$ with the experimental results of Liu et al [147] and Chen et al [148]. This implies the $\mathrm{Nu}$ augmentation to be more due to the mixing rendered by essentially the longitudinal vortices gaining strength with increase in Re than by boundary layer interruptions. All these analyses suggest improvement over smooth channel performance, but lack relative comparison of the LVG and fin structures, where boundary layer interruption remains the chief mechanism of performance enhancement. Ebrahimi et al [149] found that the combination of converging and diverging angles of vortex generators improve the performance of the channel. Between the aligned and staggered combinations of LVGs

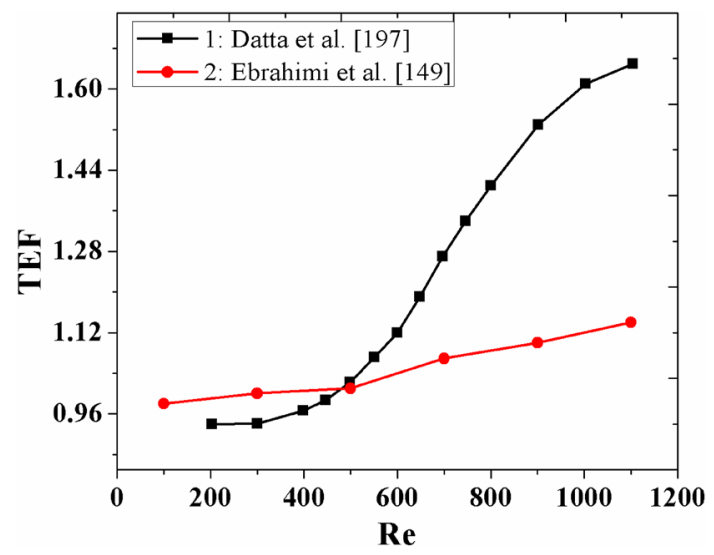

Figure 10. Optimal TEF predictions for channel with LVG for 1: more than two pairs of vortex generators; 2 : two pairs of vortex generator.

of five and ten pairs placed over an axial length of $20 \mathrm{~mm}$ studied by Datta et al [197], the maximum TEF of 1.6 was predicted for ten aligned pairs of LVGs at $30^{\circ}$ angle. They found that the vortices from VGs following the first set near the entrance increase the heat transfer more. In the investigated range of Re from 200 to 1200, TEF exhibited an increasing trend with increase in Re with indications of saturation towards higher Re. With further increase in LVG angles from $30^{\circ}$, the accompanying rise in $\mathrm{f}$ due to higher flow obstruction negates the rise in $\mathrm{Nu}$, thereby bringing down the performance. The improvement of TEF with LVG is shown in figure 10. Another effect of higher LVG inclination is better mixing due to stronger secondary flow arising from higher flow turning. The aspect of flow turning has been investigated extensively in zigzag and serpentine microchannels in terms of chaotic advection.

Microchannels with VGs should be configured with channel width greater than the height. The recommended height of the VGs is lower than the channel height to aid formation of LVGs. These vortices significantly enhance the heat transfer at low Re for their domain of travel churning through the flow being much larger than transverse vortices that approach the near-by side wall too quickly and dump most of the energy there. Appropriate combination of diverging and converging angles of VGs gives higher performance. The authors came across the shape of VGs as rectangular only in all significant investigations pertaining to microchannels. Prior to considering the technology challenge, other innovative VG shapes warrant a prior numerical assessment of their performance.

\section{Channels with converging-diverging side walls or curved axis}

In a general survey of heat transfer enhancement techniques pertaining to single-phase flow through mini- and microchannels, Steinke and Kandlikar [151] identified 


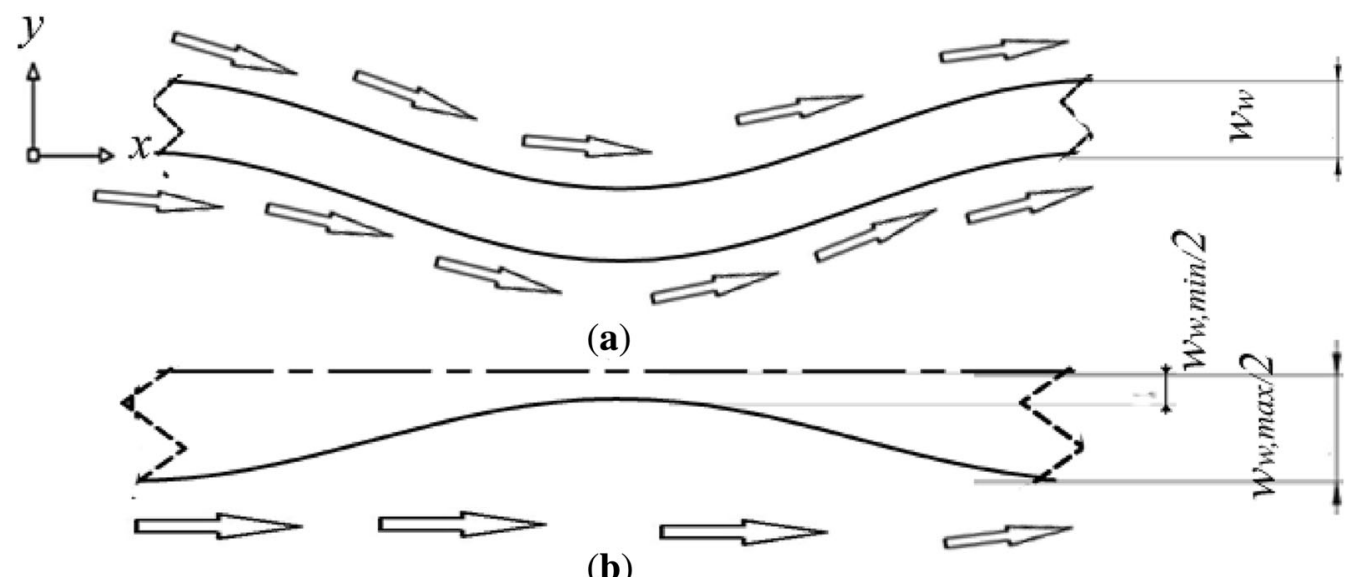

(b)

Figure 11. An intermediate side wall for $(a)$ serpentine channel, $(b)$ raccoon channel.

curved channels as the most practical solution. Biswas et al [81] and Ghani et al [67] provide excellent reviews on flow and heat transfer in wavy micochannels of zigzag, circular and sinusoidal forms of constant flow area broadly referred to as serpentine channels. These are becoming attractive for their potential to enhance the heat transfer together with keeping the pressure drop relatively lower than that in corresponding straight channel of identical cross section and footprint length. Chu et al [152, 153] established the applicability of conventional laminar flow analysis through experimental validation in terms of friction factor for different curvature and aspect ratios of rectangular microchannel over Re range of 10 to 876 . At higher Re, Yang et al [154] obtained good agreement with experimental results by using roughness viscosity model for the wall.

Sui et al $[155,156]$ observed the maximum pressure at the outer wall and the minimum at the inner wall of a wavy channel. The centrifugal force gets balanced by the pressure gradient generating a pair of counter rotating vortices across the flow. These vortices in opposite rotation are known as Dean vortices, which cause substantial rise in convective heat transfer with minimal enhancement in pressure drop along the dominant flow direction. At higher $\mathrm{Re}$, the Dean vortices promote chaotic advection and hence the heat transfer through invigorated flow mixing. The shapes and axial position of the vortices too depend on Re. Sui et al [155] successfully compared experimental results with their numerical predictions over $0-259 \mu \mathrm{m}$ range of wavy amplitude and $2.5 \mathrm{~mm}$ wavelength. A numerical study by Sui et al [156] indicated increase in the heat transfer over pressure drop for decrease in the channel wavelength. Thus, the wavelength could be adapted to locally different cooling needs for different electronic chips deployed on the same circuit board, thereby avoiding possible hot spots from developing in regions of high heat flux.

Another geometrical configuration for imparting curvature to the flow that is being studied is an arrangement of alternating back-to-back multiple units of converging-diverging sections with channel axis curved or straight. A straight-axis channel with converging-diverging section is also referred to as raccoon channels. The shapes of the intermediate side walls in serpentine and raccoon channels are indicated in figure 11. Duryodhan et al [157-159] performed experiments involving flow through straightwalled straight-axis microchannels of single unit of converging, diverging or converging-diverging passage under relatively low wall heat flux in the range of 0.3 to 9.5 $\mathrm{W} / \mathrm{cm}^{2}$. The last one of these three studies clearly established the necessity of conjugate heat transfer modeling for numerical analysis. While a converging passage revealed significant contribution to enhancing the heat transfer, the flow divergence below $12^{\circ}$ was found to reduce the pumping power to even one-fifth relative to that in a channel with uniform flow section. Thus, a combined converging-diverging passage was concluded as an effective arrangement for improving the TEF. Gong et al [160] predicted the TEF of sinusoidal serpentine channels to be significantly superior to raccoon channels with a straight axis. The dependence of TEF on the geometry of the wavy wall keeps its research interest alive.

Numerical results of Gong et al [160] and Ghaedemini et al [161] showed the TEF in sinusoidal raccoon channels with straight axis to be lower than in serpentine channels chiefly due to the gain in $\mathrm{Nu}$ undone by the sharp rise in $\mathrm{f}$ worsening with increase in wavelength. Streamline patterns in raccoon channels constructed by Yong et al [162] from their numerical results showed good agreement with laser sheet flow visualization results for Re 100 and 200. The numerical results for Re 50,100, 150 and 200 revealed the TEF for sinusoidal waveform to be in general superior to circular waveform characterized by constant curvature, all revealing saturation beyond waviness higher than 0.05 . Their numerical predictions also exhibited close matching with the predictions by Gong et al [160]. 
For Re of 200, 400 and 600, Ghaedemini et al [161] found the initially rising trend in TEF from 1.0 to a nominally higher value of 1.2 for increase in waviness from 0 to 0.05 reverting to a falling trend for further increase in waviness showing the TEF to go down to 0.2 for channel aspect ratio 1.5 and waviness 0.15 . The scenario showed some improvement with increase in Re and change of aspect ratio in either direction. Gong et al [160] noticed rise of TEF with increase in waviness for Re 100 and 150 possibly from increase in local $\mathrm{Nu}$ in the high-velocity, low-area flow passage. Dharaiya and Kandlikar [163] predicted spikes in wall temperature for sinusoidal raccoon microchannels. The numerical results of Ma et al [164] in raccoon channels with zigzag side walls exhibited the possibility of achieving TEF as high as about 1.6 with expanding length occupying only about $10 \%$ of a raccoon unit. In fact, $f$ for such a shape was found to be lower than that in a smooth channel.

In the Re range of 50-150 pertaining to serpentine channels, the results of Gong et al [160] revealed progressively increasing rise of TEF with increase in amplitude getting stronger at higher Re. No significant dependence of TEF was observed on wavelength in the range 1 to $4 \mathrm{~mm}$ or on wave amplitude below $50 \mu \mathrm{m}$ or on any wave amplitude for Re equal to 50. Mohammed et al [165] predicted the highest heat transfer coefficient for parallel arrangement of 14 to 16 channels depending on the sinusoidal waviness value. One of their significant finding is the gradual increase in both $\mathrm{Nu}$ and $\mathrm{f}$ with increase in channel waviness from 0 to 0.21875 contrasted by a sudden increase in $\mathrm{f}$ together with decrease in $\mathrm{Nu}$ going even below to that of the straight channel for a value of 0.25 . Alam and Kim [166] considered grooves along the circular bends of serpentine microchannels in the Re range of 0.5 and 90 and found the groove width to have more significant effect than the groove depth. A numerical study by Sakanova et al [167] showed insignificant effect of adding nanoparticles to flowing water through microchannels of sinusoidal waveform. Chiam et al [168] conducted a combined numerical and experimental study for sinusoidal curved microchannels with secondary channel interruptions at the troughs and crests of the wavy channels for Re range 50-200 and relative amplitude of 0.15 and 0.075 , where the relative amplitude is defined as the ratio of the channel amplitude to wavelength of a wavy unit. It is interesting to note that the performance enhancement is achieved in channels with increase and decrease of wavy amplitude, respectively for channels without and with secondary branches $[156,168]$. The secondary channels were found to be more effective in raising the TEF at lower Re and lower relative amplitude [168]. It is understandable that lower value of both these parameters entail weaker Dean rolls pushing lesser flow through the secondary channels. This results in lower loss of momentum in the primary channel, in turn reflected as not so poor pressure recovery in comparison to the wavy design without secondary channels. Hot spots are less likely in case of higher momentum in the primary channel for the lower values of both the factors. An interesting outcome of this study is finding an arrangement of better thermal management with lower flow. This study requires greater attention with deployment of different designs of secondary channels and wider parameter ranges.

In curved flow, an important mixing mechanism is chaotic advection that evolves from a secondary flow pattern as Re crosses a critical value. Aref [169, 170], Ottino [171], Stroock et al [172], Schonfeld and Hardt [173] and Jiang et al [174] discussed the potential of chaotic advection for augmenting mixing in two-dimensional unsteady or three-dimensional flows. Kalb and Seader [175], Masliyah and Nandakumar [176], Yang et al [177] and Wang and Yang [178] discussed its positive role in enhancing heat transfer as well. Analysis tools like Poincare section and fast Fourier transform, or FFT, have been used to investigate the extent of chaotic advection. Sui et al [155, 156, 179], Zheng et al [180] and Abed et al [181] constructed Poincare sections and Sui et al [179] used FFT, all on their numerical results. Incidentally, the investigations by Sui et al [156] and Abed et al [181] on sinusoidal and circular wavy channels involve experimental results as well for the validation of numerical predictions of $\mathrm{Nu}$ and $\mathrm{f}$.

Zheng et al [180] and Abed et al [181] found chaotic mixing to set in above certain Re in their studies with ten consecutive units, with each unit containing two successive bends of opposite curvatures. Besides $\mathrm{Nu}$ variation being proportional to one-third power of Prandtl number, Zheng et al [180] also predicted a spatially periodic flow to become chaotic with increase in Re beyond 200 in their study on zigzag bends of semi-circular cross section. Beyond a threshold, the wall boundary layers cannot sustain the inward radial pressure gradient arising from the flow turning. As a result, a secondary flow sets in along the transverse plane. Dean $[182,183]$ was first to provide an analytical solution for this flow in a pipe bend giving rise to a pair of rolling vortices, radially outward in the vicinity of the mid plane and inward along the facing walls. This pair of symmetric counter-rotating vortices is popularly referred to as Dean rolls that provide an additional mechanism of flow mixing. Sugiyama et al [184] and Mokrani et al [185] observed the rolling to get associated with one more vortex on each side of the mid plane referred to as Dean vortices. The flow visualization experiments of Sugiyama et al [184] revealed that these rolls and vortices acquire locations which are strongly dependent on the channel aspect ratio rather than Dean number. The Dean number is defined as

$$
\mathrm{DN}=\operatorname{Re} / \sqrt{w / R},
$$

where $w$ and $R$ are the width of the mid plane and the mean radius of the channel bend.

Numerical results at low Re deserve a careful look. Xie et al [186, 187] found both rectangular and transversely wavy flow sections with straight axis to have better TEF 
than a wavy serpentine channel. This must have arisen from studied low ranges for both Re and waviness. Gong et al [160] observed secondary flow to improve heat transfer even in the absence of Dean vortices. A combined numerical and experimental study by Jiang et al [174] for flow through curved rectangular microchannel confirmed chaotic mixing to begin beyond a DN of around 100. The numerical study of Facao and Oliviera [188] revealed up to a ten-fold increase in $\mathrm{Nu}$ due to curvature of a rectangular channel along with splitting of the rolling pair to three vortex pairs at DN of 131. Similar investigations by Rosaguti et al [189-191] and Geyer et al [192] showed two and half to three times rise in $\mathrm{Nu}$ for flow of water through wavy microchannels depending on whether the flow section is circular, semi-circular or square. Complex vortex patterns with more than one pair were also reported. Of course, a vortex pattern of greater complexity forces a material point to trace a more tortuous path line that are vividly portrayed by Poincare sections.

A Poincare section contains points on the path lines downstream of an initial section having a set of chosen close by tracer particles. The path line for each tracer is estimated say by Runge-Kutta time integration of the particle velocity estimated at its convected locations by interpolating the neighbouring point velocities computed on an Eulerian frame say by the control volume method. Often a set of Poincare sections at specific intervals are merged together as one. Thus a visual is constructed showing the extent of stretching, folding and dispersion of connected material points inflicted by the flow.

Zheng et al [180] and Abed et al [181] considered the tracer points lying initially on a vertical line at the mean channel radius on the horizontal plane. Abed et al [181] constructed Poincare sections for the rectangular channel after ten serpentine circular bends from the inlet section for increasing DN of $0.65,42.6$ and 106.5 corresponding to $\mathrm{Re}$ of about $0.53,52$ and 130 in their study. No significant variation of particle positions relative to the inlet for the lowest DN implies the consequent pressure gradient as insufficient for generating the secondary flow. At the intermediate DN, the Poincare section is consistent to a pair of structured Dean rolls with axis of each roll hovering in a zone away from the walls and the horizontal mid span. This is evident from the higher stretching of the elements initially near the mid span and either the top or bottom wall relative to the elements in between. At the highest DN, the particles appear as well dispersed without crossing over to the other side of the horizontal mid span. This dispersion is due to the breakdown of the pair of vortices to smaller multiple vortices in each half, forcing two nearby particles to follow distinctly different paths by two adjacent vortex structures. Even more chaotic pattern at higher Re of 200 and 300 are predicted in a semi-circular channel section by Zheng et al [180] that are evident from the merged Poincare sections at the inlet of the seventh, eighth and ninth units for a set of particles initially at the inlet of the sixth unit.
A notable feature of the Poincare section constructed by Sui et al [155] for the horizontal element at the mid span of the exit of the fifth sinusoidal unit of a rectangular channel is the progressively increasing stretch at the exits of four subsequent units spreading across both sides of the mid span for $\operatorname{Re}$ of 100 . This contrasts the relatively weaker stretching and dispersion of each particle on a vertical element remaining confined to the half of the initial section for even a higher Re of about 130 obtained by Abed et al [181] for a serpentine waveform. Therefore, the sinusoidal waviness of a channel can be regarded as superior to the serpentine form. Figure 12 shows the TEF variation calculated from the data of Sui et al [156]. Among these studies, the sinusoidal channel shows the best TEF. The significant observations related to converging diverging channel are listed in table 7.

A study by Gong et al [160] in a similar channel form revealed: (1) progressively increasing rise of TEF with increase in amplitude getting stronger at higher $\mathrm{Re}$, (2) no significant rise of TEF with increase in wave amplitude either for Re below 50 or for wave amplitude below $50 \mu \mathrm{m}$, and (3) lower effect of change in wavelength and channel aspect ratio on dispersion. Mohammed et al [85] predicted the highest heat transfer coefficient for an amplitude-ratio dependent arrangement of 14 to 16 parallel sinusoidal channels.

Contrasting the mainstream steady flow analysis, Sui et al [179] carried out an unsteady simulation within the conventional laminar incompressible flow and conjugate heat transfer framework. They investigated flow through a serpentine microchannel bend of rectangular section. At Re of 66.67, the flow was predicted as steady proceeding along helical path lines for being associated with a pair of symmetric Dean rolls. With increase in Re, the centres of the rolls exhibited shift away from the streamline parting the pairs. For a higher Re of 166.7, one more pair of symmetric steady vortices appeared providing much wider dispersion

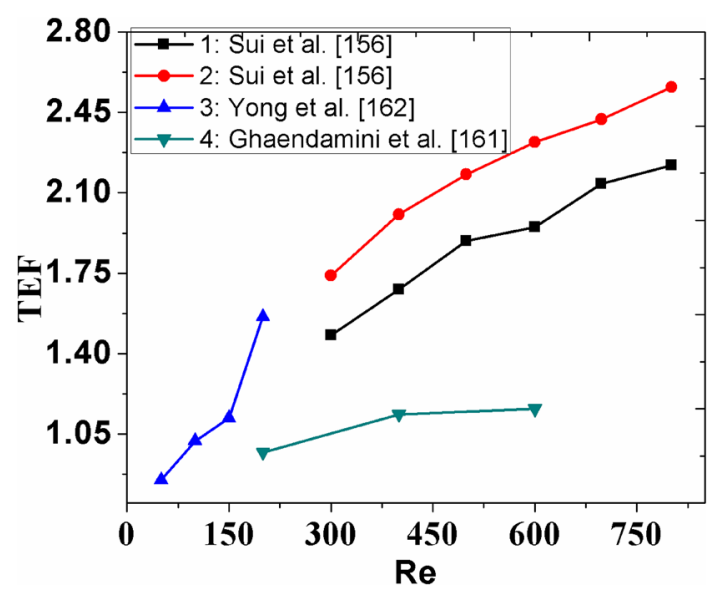

Figure 12. TEF predictions for microchannels with convergingdiverging side walls or curved axis with legends explained in the last column of table 7 . 


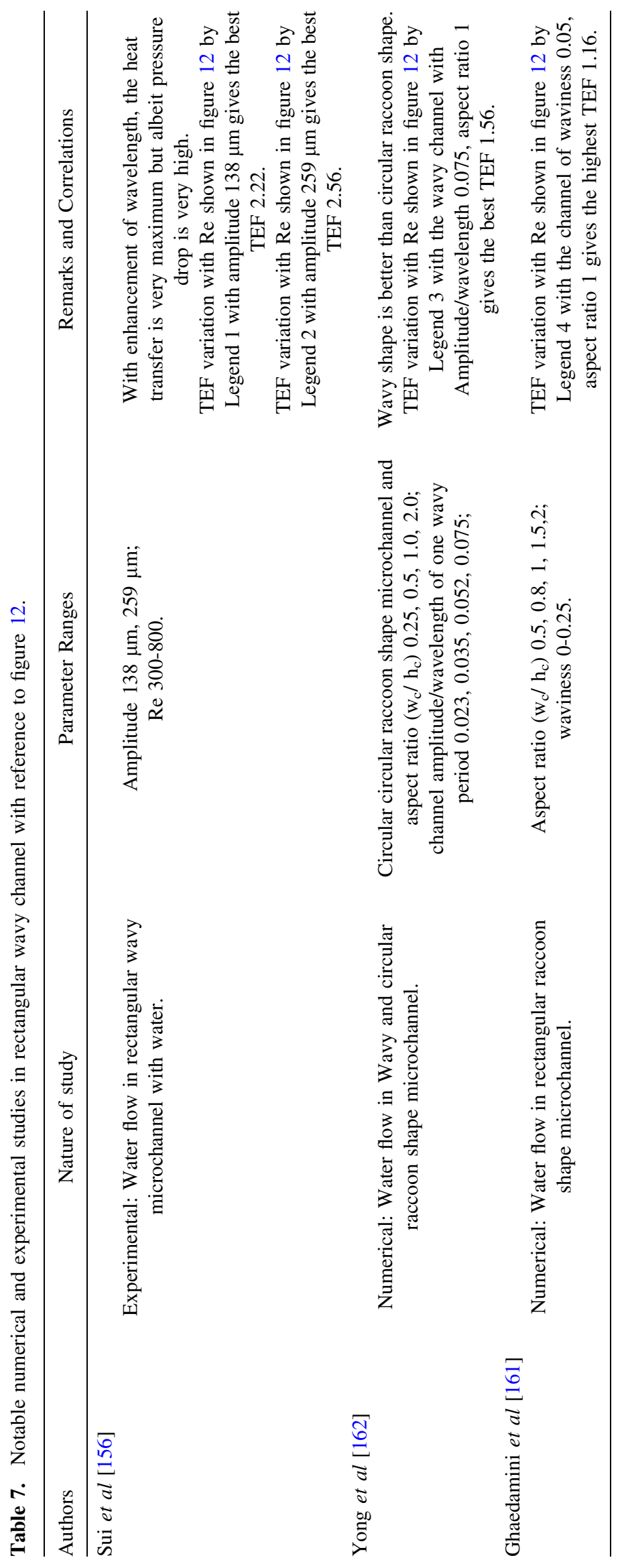


of particles, as revealed by the Poincare maps. Results at Re of 233.3 indicated further strengthening of the mixing mechanism due to the periodic oscillation of all the four vortex structures both in time and space. The FFT of evolution of the velocity on the channel axis at the mid length revealed a single-frequency periodicity at $\operatorname{Re}$ of 266.7 together with loss of symmetry exhibited by the velocity vector plots. A transition to two-frequency quasi-periodic regime through Hopf bifurcation was evident at $\mathrm{Re}$ of 333.3. Beyond $\operatorname{Re}$ of 366.7 , fully aperiodic structure emerged signalling the onset of chaotic advection. Fully developed velocity profile at the inlet was considered in order to set aside the entrance region effects on the study. However, such effects cannot be ignored especially in the earlier bends in real applications.

Wang and Liu [193] used the transient form of the conventional laminar-flow model for a curved channel of constant curvature and square section to analyze the flow bifurcation effect of the initial conditions and finite disturbances. With increase in DN from 0 to 2250, the solution showed existence of two symmetric and eight asymmetric branches along with thirty one limit points. Progression from a stable steady two-cell state through multiple oscillating cells to a chaotic temporal oscillation was captured. A drastic change in $\mathrm{Nu}$ with initiation of flow oscillation was predicted. The effect of channel curvature was seen to enhance $\mathrm{Nu}$ at the cost of marginal increase in $\mathrm{f}$. Experiments of Dai et al [194, 195] indeed revealed significant effect of inlet condition on chaotic advection in microchannels of semi-circular cross section of zigzag or sinusoidal waveforms. Hasis et al [196] carried out a numerical study in a twisted rectangular microchannel. Higher heat transfer was predicted for a channel of higher aspect ratio and lower waviness and with respect to an untwisted wavy channel; 30\% enhancement was observed in the low Re range.

\section{Conclusions}

The recent trend exhibited by the pertinent research is to use conventional laminar-flow conjugate heat-transfer modeling with no-slip flow at the walls with prior validation against experimental results for simple situations with follow-up advanced experiments for potential situations revealed by the experimental studies. Of course, the judicial use of sophisticated experimental resources has been made in view of the challenges that arise from very tiny characteristic dimensions and intricacies of the flow passages. The severity of the challenges is evident from the gross uncertainties associated with the earlier experimental results and interpretations. A number of experimentalists interpreted the increased sensitivity of Nusselt number rise with Reynolds number as an early onset of flow transition from laminar to turbulent regime. It is quite possible that the observed higher degree of sensitivity is due to the extension of the entrance region with increase in Reynolds number. Indeed, such beneficial effect of entrance region flow is exploited by inserting flow disruptions in microchannels. However, this benefit needs to be weighed against the penalty of increased pressure loss.

Flow disruption and chaotic advection have been identified as the two chief heat transfer enhancement mechanisms in the context of single-phase flow of simple liquids that are expected to dominate the mainstream research for improving cooling performance in electronic devices for some time to come. Boundary layer interruption at the edges of the disruptive structures causes sharp rise of local heat transfer coefficients. The review includes two-dimensional ribs cavities, fins as well as three dimensional dimples and protrusions along with their different combinations in an otherwise straight microchannel. A review has also been made for the emerging trend of microchannel studies with wavy axis.

As expected, two-dimensional structures provide disruptions at larger scale with higher penalty of pressure drop. Additional flow mixing arises from other structural forms by either shedding of vortices from the trailing edges of longitudinal vortex generators or by homogenizing the main channel flow by cross transport through slanted secondary channels. Keeping the microchannel dimensions in mind, most of the vortex generator studies considered symmetrically arranged array of inclined rectangular plates across the channel depth. The beneficial effect of interruption of the main channel flow by in-between transverse channels has also been investigated. For augmenting TEF in microchannels with VGs, higher width has been observed to be more beneficial than higher channel height. A VG extended over the entire channel height blocks the possibility of shedding longitudinal vortices through the gaps at the top and bottom of a VG. The resultant transverse vortices waste a large amount of momentum on the side walls, whereas the longitudinal vortices cause good flow mixing and consequent enhancement in the heat transfer over a longer part of the domain.

A cavity, dimple or microchamber causes a local flow expansion while a rib, fin, or protrusion gives rise to a local flow contraction. Of course, an inclined VG makes the flow expanding at one side and contracting on the other side. Experimental studies revealed heat transfer enhancement in the contracting portions characterized by accelerated flow and pressure recovery in the expanding parts entailing flow deceleration. Hence, a trend of combining the flow expanding and the contracting elements has dominated the microchannel studies so as to arrive at an efficient thermal management design. Among the different disruptive structures, rib within the cavity from the bottom wall is found to be quite effective in improving the TEF. The advancement of the microchannel fabrication technology for having ellipsoidal ribs or curved VGs and the development of nonlinear global multi-parameter optimizers keep this 
branch of constructional optimization live. Research by Adham et al [10] and Datta et al [197] are the early steps for the optimization studies in the context of microchannels.

The current study reveals that the secondary channel in the side wall of channel has shown promise in achieving high TEF especially at low Re. Their use together with the primary channels with expanders and constrictions has however found scant attention. Investigation on such combinations is therefore a recommended direction for further work. As far as enhancing heat transfer without significant rise in the pressure loss is concerned, the mechanism of chaotic advection associated with curving flow is considered as superior to flow disruptions. Available results from the numerical studies are indicative of that despite the much richer extent of studies by geometric manoeuvring for promoting flow disruptions. Hence, investigation of curving flow by newer channel design concepts demands higher attention than that it has received so far. The heat transfer in curved channel flow keeps increasing with Reynolds number due to flow patterns from a pair of steady rolling secondary vortices in the transverse plain changing to oscillating multiple pairs of vortices ultimately giving rise to chaotic patterns. These have been established by Poincare sections and bifurcation studies. Combination of secondary channels with wavy primary channel of different waviness at different regions has been identified to be quite promising, especially for achieving high TEF at low Re and thermal management with disparate cooling requirements at different parts of the electronic substrate.

\section{Nomenclature}

\section{Symbol}

$b \quad$ base width of roughness

$d_{h} \quad$ hydraulic diameter

$D$ depth of dimple or height of protrusion

DN Dean number

f friction factor

$h_{c} \quad$ height of channel

$h_{r} \quad$ roughness height

$l_{c} \quad$ height of channel

$\mathrm{Nu} \quad$ Nusselt number

Pr Prandtl number

$p \quad$ longitudinal spacing or pitch

Re Reynolds number

$\operatorname{Re}_{c, f}$ critical $R e$ across which slope of $f$ variation with $R e$ changes sharply

$s \quad$ transverse spacing

TEF thermal enhancement factor

$w_{b} \quad$ bottom width of trapezoidal channel

$w_{c} \quad$ top width or uniform width of channel

$w_{w} \quad$ width of channel wall

\section{Subscripts}

0 smooth channel

\section{References}

[1] Tuckerman D B and Pease R F W 1981 High-performance heat-sinking for VLSI. IEEE Electron Device Letters 2: 126-129

[2] Ghaedamini H, Lee P S and Teo C J 2013 Developing forced convection in converging-diverging microchannels. Int. J. Heat Mass Transfer 65: 491-499

[3] Walker J L 2011 Handbook of RF and Microwave Power Amplifiers. Cambridge, UK: Cambridge University Press

[4] Krishnan S, Garimella S V, Chrysler G M and Mahajan R V 2007 Towards a thermal Moore's law. IEEE Trans. Adv. Packag. 30: 462-474

[5] Chai L, Xia G, Wang L, Zhou M and Cui Z 2013 Heat transfer enhancement in microchannel heat sinks with periodic expansion-constriction cross-sections. International Journal of Heat and Mass Transfer 62: 741-751

[6] Kandlikar S G and Grande W J 2003 Evolution of microchannel flow passages- thermohydraulic performance and fabrication technology. Heat Transfer Engineering 24: 3-17

[7] Mehendale S S, Jacobi A M and Shah R K 2000 Fluid flow and heat transfer at micro-and meso-scales with application to heat exchanger design. Trans. ASME Applied Mechanics Reviews 53: 175-193

[8] Agrawal A, Kushwaha H M, Jadhav R S 2019 Microscale flow and heat transfer: mathematical modelling and flow physics. Springer.

[9] Webb R L 1981, Performance evolution criteria for use of enhanced heat transfer surfaces in heat exchanger design. International Journal of Heat and Mass Transfer 24: 715-726

[10] Adham A M, Mohd-Ghazali N and Ahmad R 2013 Thermal and hydrodynamic analysis of microchannel heat sinks: A review. Renewable and Sustainable Energy Reviews 21: 614-622

[11] Kaushik K, Pati S, Som S K and Chakraborty S 2012 Hydrodynamic and thermal transport characteristics of swirling flows through microchannels with interfacial slip. International Journal of Heat and Mass Transfer 55: 4359-4365

[12] Shkarah A J, Sulaiman M, Ayob M and Togun H 2013 A 3D numerical study of heat transfer in a single-phase microchannel heat sink using graphene, aluminum and silicon as substrates, International Communications in Heat and Mass Transfer 48: 108-115

[13] Hung T C, Huang Y X and Yan W M 2013 Thermal performance analysis of porous microchannel heat sinks with different configuration designs. International Journal of Heat and Mass Transfer 66: 235-243

[14] Hung T C, Huang Y X and Yan W M 2013 Thermal performance of porous microchannel heat sink: effects of enlarging channel outlet. International Communications in Heat and Mass Transfer 48: 86-92

[15] Farsad E, Abbasi S P and Zabihi M S 2014 Fluid flow and heat transfer in a novel microchannel heat sink partially 
filled with metal foam medium. Trans. ASME Journal of Thermal Science and Engineering Applications 6: 021011:1-7, 2014

[16] Shen B, Yan H, Sunden B, Xue H and Xie G 2017 Forced convection and heat transfer of water-cooled microchannel heat sinks with various structured metal foams, International Journal of Heat and Mass Transfer 113: 1043-1053

[17] Calmidi V and Mahajan R 2000 Forced convection in high porosity metal foams. Trans. ASME Journal of Heat Transfer 122: 557-565

[18] Phillips R J 1988 Microchannel heat sinks. The Lincoln Labortory Journal 1: 31-47.

[19] Kandlikar S G and Grande W J 2004 Evaluation of single phase flow in microchannels for high heat flux chip cooling - thermohydraulic performance enhancement and fabrication technology. Heat Transfer Engineering 25: 5-16

[20] Dixit T and Ghosh I 2015 Review of micro- and minichannel heat sinks and heat exchangers for single phase fluids. Renewable and Sustainable Energy Reviews 41: 1298-1311

[21] Croce G and D'Agaro P 2005 Numerical simulation of roughness effect on microchannel heat transfer and pressure drop in laminar flow. Journal of Physics D 38: 1518-1530

[22] McHale J P and Garimella S V 2010 Heat transfer in trapezoidal microchannels of various aspect ratios. International Journal of Heat and Mass Transfer 53: 365-375

[23] Gunnasegaran P, Mohammed HA, Shuaib N H and Saidur R 2010 The effect of geometrical parameters on heat transfer characteristics of microchannel heat sink with different shapes. International Communications in Heat and Mass Transfer 37: 1078-1086

[24] Morini G L 2004 Laminar-to-turbulent flow transition in microchannels. Microscale Thermophysical Engineering 8: $15-30$

[25] Morini G L 2004 Single-phase convective heat transfer in microchannels: a review of experimental results. International Journal of Thermal Sciences 43: 631-651

[26] Zhuo L, Tao W and Ya-Ling H 2006 A numerical study of laminar convective heat transfer in microchannel with noncircular cross-section. International Journal of Thermal Sciences 45: 1140-1148

[27] Wu H and Cheng P 2003 Friction factors in smooth trapezoidal silicon micro-channels with different aspect ratios. International Journal of Heat and Mass Transfer 46: 2519-2525

[28] Sadasivam R, Manglik R M and Jog M A 1999 Fully developed forced convection through trapezoidal and hexagonal ducts. International Journal of Heat and Mass Transfer 42: 4321-4331

[29] Saha S K, Agrawal A and Soni Y 2017 Heat transfer characterization of rhombic microchannel for $\mathrm{H} 1$ and $\mathrm{H} 2$ boundary conditions. International Journal of Thermal Sciences 111: 223-233

[30] Wang B X and Peng X F 1994 Experimental investigation on liquid forced-convection heat transfer through microchannels. International Journal of Heat and Mass Transfer 37: 73-82

[31] Peng X F and Peterson G P 1996 Convective heat transfer and friction for water flow in micro-channel structures. International Journal of Heat and Mass Transfer 39: 2599-2608
[32] Qu W, Mala G M and Li D 2000 Heat Transfer for water flow in trapezoidal silicon microchannels. International Journal of Heat and Mass Transfer 43: 3925-3936

[33] Hetsroni G, Mosyak A, Pogrebnyak E and Yarin L P 2005 Fluid flow in micro-channels. International Journal of Heat and Mass Transfer 48: 1982-1998

[34] Valdes J R, Miana M J, Pelegay J L, Nunez J L and Putz T 2007 Numerical investigation of the influence of roughness on the laminar incompressible fluid flow through annular microchannels. International Journal of Heat and Mass Transfer 50: 1865-1878

[35] $\mathrm{Hu} \mathrm{Y,} \mathrm{Werner} \mathrm{C} \mathrm{and} \mathrm{Li} \mathrm{D} 2003$ Influence of threedimensional roughness on pressure-driven flow through microchannels. Journal of Fluids Engineering 125: 871-879

[36] Rawool A S, Mitra S K and Kandlikar S G, Numerical simulation of flow through microchannels with designed roughness. Microfluidics and Nanofluidics 2: 215-221

[37] Croce G, D'agaro P and Nonino C 2007 Three-dimensional roughness effect on microchannel heat transfer and pressure drop. International Journal of Heat and Mass Transfer 50: 5249-5259

[38] Gamrat G, Favre-Marinet M and Person S L 2009 Modelling of roughness effects on heat transfer in thermally fully-developed laminar flows through microchannels. International Journal of Thermal Sciences 48: 2203-2214

[39] Wu H and Cheng P 2003 An experimental study of convective heat transfer in silicon microchannels with different surface conditions. International Journal of Heat and Mass Transfer 46: 2547-2556

[40] Harms T M, Kazmierczak M J and Gerner F M 1999 Developing convective heat transfer in deep rectangular microchannels. International Journal of Heat and Fluid Flow 20: 149-157

[41] Xu B, Ooti K T, Wong N T and Choi W K 2000 Experimental investigation of flow friction for liquid flow in microchannels. International Communications in Heat and Mass Transfer 27: 1165-76

[42] Judy J, Maynes D and Webb B W 2002 Characterization of frictional pressure drop for liquid flows through microchannels. International Journal of Heat and Mass Transfer 45: 3477-3489

[43] Qu W and Mudawar I 2002 Experimental and numerical study of pressure drop and heat transfer in a single-phase micro-channel heat sink. International Journal of Heat and Mass Transfer 45: 2549-2565

[44] Liu D and Garimella S V 2004 Investigation of liquid flow in micro channels. AIAA Journal of Thermo Physics Heat Transfer 18: 65-72

[45] Liu Y, Cui J, Li W Z and Zhang N 2011 Effect of surface microstructure on microchannel heat transfer performance. ASME Journal of Heat Transfer 133: 124501

[46] Mansoor M M, Wong K and Siddique M 2012 Numerical investigation of fluid flow and heat transfer under high heat flux using rectangular micro-channels. International Communications in Heat and Mass Transfer 39: 291-297

[47] Lee P, Garimella S V and Liu D 2005 Investigation of heat transfer in rectangular microchannels. International Journal of Heat and Mass Transfer 48 (9): 1688-1704

[48] Tiselj I, Hetsroni G, Mavko B, Mosyak A, Pogrebnyak E and Segal Z 2004 Effect of axial conduction on the heat 
transfer in micro-channels. International Journal of Heat and Mass Transfer 47: 2551-2565

[49] Lee P and Garimella S V 2006 Thermally developing flow and heat transfer in rectangular microchannels of different aspect ratios. International Journal of Heat and Mass Transfer 49: 3060-3067

[50] Mishan Y, Mosyak A, Pogrebnyak E, and Hetsroni G 2007 Effect of developing flow and thermal regime on momentum and heat transfer in micro-scale heat sink. International Journal of Heat and Mass Transfer 50: 3100-3114

[51] Xu J, Song Y, Zhang W, Zhang H and Gan Y 2008 Numerical simulations of interrupted and conventional microchannel heat sinks. International Journal of Heat Mass Transfer 51: 5906-5917

[52] Gulhane N P and Mahulikar S P 2011 Numerical study of microconvective water-flow characteristics with variations in properties. Nanoscale and Microscale Thermophysical Engineering 15: 28-47

[53] Gulhane N P and Mahulikar S P 2012 Numerical investigation on laminar microconvective liquid flow with entrance effect and Graetz problem due to variation in thermal properties. Heat Transfer Engineering 33: 748-761

[54] Kumar R and Mahulikar S P 2015 Effect of temperaturedependent viscosity variation on fully developed laminar micro convective flow. International Journal of Thermal Sciences 98: 179-191

[55] Moharana M K, Singh P K and Khandekar S 2011 Axial heat conduction in the context of developing flows in microchannels. In: ASME 2011 9th International Conference on Nanochannels, Microchannels, and Minichannels. American Society of Mechanical Engineers, pp. 455-462

[56] Moharana M K and Khandekar S 2012 Effect of channel shape on axial back conduction in the solid substrate of microchannels. In: Proceedings of the 3rd European conference on Microfluidics-Microfluidics, 2012-Heidelberg

[57] Rahimi M and Mehryar R 2012 Numerical study of axial heat conduction effects on the local Nusselt number at the entrance and ending regions of a circular microchannel. International Journal of Thermal Sciences 59: 87-94

[58] Nonino C, Savino S and Del Giudice S 2010 Temperaturedependent viscosity and viscous dissipation effects in microchannel flows with uniform wall heat flux. Heat Transfer Engineering 31: 682-691

[59] Kong K S and Ooi K T 2013 A numerical and experimental investigation on microscale heat transfer effect in the combined entry region in macro geometries. International Journal of Thermal Sciences 68: 8-19

[60] Liu Z, Zhang C, Huo Y and Zhao X 2007 Flow and heat transfer in rough micro steel tubes. Experimental Heat Transfer 20: 289-306

[61] Jian M, Yan P H, Jun H, Yan L W and Qiu W W 2010 Experimental investigations on single-phase heat transfer enhancement with longitudinal vortices in narrow rectangular channel. Nuclear Engineering and Design 240: 92-102

[62] Peng X F and Peterson G P 1995 The effect of thermofluid and geometric parameters on convection of liquid through rectangular microchannels. International Journal of Heat and Mass Transfer 38: 755-758
[63] Peng X F and Peterson G P 1995 Frictional flow characteristics of water flowing through rectangular microchannels. Experimental Heat Transfer an International Journal 7 (4): 249-264

[64] Pfund D, Rector D and Shekarriz A 2000 Pressure drop measurements in a micro-channel. AIChE Journal 46: 1496-1507

[65] Maynes D and Webb A R 2002 Velocity profile characterization in sub-diameter tubes using molecular tagging velocimetry. Experiments in Fluids 32: 3-15

[66] Hao P, He F,and Zhu K 2005 Flow characteristics in a trapezoidal silicon microchannel Journal of Micromechanics and Microengineering 15:1362-1368

[67] Ghani I A, Sidik N A C and Kamaruzaman N 2017 Hydrothermal performance of microchannel heat sink: The effect of channel design. International Journal of Heat and Mass transfer 107: 21-44

[68] Ebadian M A and Lin C X 2011 A review of high-heat-flux heat removal technologies. Journal of Heat Transfer 133: $1-11$

[69] Boyd R D 1985 Subcooled flow boiling critical heat flux (CHF) and its application to fusion energy components. Part 1. A review of fundamentals of $\mathrm{CHF}$ and related data base. Fusion Technology 7: 7-30

[70] Lee J and Mudawar I 2009 Low-temperature two-phase microchannel cooling for high heat-flux thermal management of defence electronics. IEEE Trans on Component Packaging and Technologies 32: 453-465

[71] Tullius J F,Vajtai R and Bayazitoglu Y 2011 A review of cooling in microchannels. Heat Transfer Engineering 32: 527-541

[72] Majumder A, Mehta B and Khandekar S 2013 Local Nusselt number enhancement during gas-liquid Taylor bubble flow in a square mini-channel: An experimental study. International Journal of Thermal Sciences 66: 8-18

[73] Kandlikar S G, Colin S, Peles Y, Garimella S V, Pease R F W, Brandner J J and Tuckerman D B 2013 Heat transfer in microchannels - 2012 status and research needs. Trans. ASME Journal of Heat Transfer 135: 091001:1-18

[74] Mudawar I 2001 Assessment of high-heat-flux thermal management schemes. IEEE Trans on Component Packaging and Technologies 24: 122-141

[75] Li W and $\mathrm{Wu} \mathrm{Z} 2010$ A general correlation for evaporative heat transfer in micro/mini-channels. International Journal of Heat and Mass Transfer 53: 1778-1787

[76] Agostini B, Fabbri M, Park J E, Wojtan L, Thome J R and Michel B 2007 State of the art of high heat flux cooling11 technologies. Heat Transfer Engineering 28: 258-281

[77] Kandlikar S G 2005 High flux heat removal with microchannels-a roadmap of challenges and opportunities. Heat Transfer Engineering 26: 5-14

[78] Kenny T W, Goodson K E, Santiago J G, Wang E, Koo JM, Jiang L, Pop E, Sinha S, Zhang L, Fogg D and Yao S, Flynn R, Chang C H and Hidrovo C H 2006 Advanced cooling technologies for microprocessor. International Journal of High Speed Electronic Systems 16: 301-313

[79] Sharma C S, Tiwari M K, Zimmermann S, Brunschwiler T, Schlottig G, Michel B and Poulikakos D 2015 Energy efficient hotspot targeted embedded liquid cooling of electronics. Applied Energy 138: 414-422 
[80] Bayraktar T and Pidugu S B 2006 Characterization of liquid flows in microfluidic systems. International Journal of Heat and Mass Transfer 49: 815-824

[81] Biswas S K, Das T and Chakraborty S 2012 Nontrivial augmentations in mixing performance through integrated active and passive mixing in serpentine microchannels. Journal of Applied Physics 111: 054904:1-10

[82] Tsia T and Chein R 2007 Performance analysis of nanofluid-cooled microchannel heat sinks. International Journal of Heat and Fluid Flow 28: 1013-26

[83] Li J and Kleinstreuer C 2008 Thermal performance of nanofluid flow in microchannels. International Journal of Heat and Fluid Flow 29: 1221-1232

[84] Bhattacharya P, Samanta A and Chakraborty S 2009 Numerical study of conjugate heat transfer in rectangular microchannel heat sink with $\mathrm{Al}_{2} \mathrm{O}_{3} / \mathrm{H}_{2} \mathrm{O}$ nano-fluid. Journal of Heat and Mass Transfer 45 (10): 1323-1333

[85] Mohammed H A, Bhaskaran G, Shuaib N H and Saidur R 2011b, Numerical study of heat transfer enhancement of counter nanofluids flow in rectangular microchannel heat exchanger. Superlattices and Microstructures 50: 215-233

[86] Mohammed HA, Gunnasegaran P,and Shuaib NH, 2010, Heat transfer in rectangular microchannels heat sink using nanofluids. Intrenational Communications in Heat and Mass Transfer 37: 1496-503

[87] Ahmed H, Mohammed H A and Yusoff M 2012 An overview on heat transfer augmentation using vortex generators and nanofluids: approaches and applications. Renewable and Sustainable Energy Reviews 16: 5951-5993

[88] Salman B H, Mohammed H A and Kherbeet A S 2012 Heat transfer enhancement of nanofluids flow in microtube with constant heat flux. International Communications in Heat and Mass Transfer 39: 1195-1204

[89] Noh N M, Fazeli A and Sidik N C 2014 Numerical simulation of nanofluids for cooling efficiency in microchannel heat sink. Journal of Advanced Research in Fluid Mechanics and Thermal Sciences 4: 13-23

[90] Abubakar S B, Sidik N A C and Ahmad A S 2016 The use of $\mathrm{Fe} 3 \mathrm{O} 4-\mathrm{H} 2 \mathrm{O} 4$ nanofluid for heat transfer enhancement in rectangular microchannel heat sink. Journal of Advanced Research in Material Science 23: 15-24

[91] Das S K, Choi S U S and Patel H E 2006 Heat Transfer in Nanofluids-A Review. Heat Transfer Engineering 27: 3-19

[92] Anoop K, Sadr R, Yu J, Kang S, Jeon S and Banerjee D 2012 Experimental study of forced convective heat transfer of nanofluids in a microchannel. International Communications in Heat and Mass Transfer 39: 1325-1330

[93] Li P, Zhang D and Xie Y 2014 Heat transfer and flow analysis of $\mathrm{Al}_{2} \mathrm{O}_{3}$ - water nanofluids in microchannel with dimple and protrusion. International Journal of Heat and Mass Transfer 73: 456-467

[94] Akbarinia A, Abdolzadeh M and Laur R 2011 Critical investigation of heat transfer enhancement using nanofluids in microchannels with slip and non-slip flow regimes. Applied Thermal Engineering 31: 556-565

[95] Toh K C, Chen X Y and Chai J C 2002 Numerical computation of fluid flow and heat transfer in microchannels. International Journal of Heat and Mass Transfer 45: 5133-5141

[96] Islami B S, Dastvareh B and Gharraei R 2013 Numerical study of hydrodynamic and heat transfer of nanofluid flow in microchannels containing micromixer. International Communications in Heat and Mass Transfer 43: 146-154

[97] Anbumeenakshi C and Thansekhar M R 2017 On the effectiveness of a nanofluid cooled microchannel heat sink under non-uniform heating condition. Applied Thermal Engineering 113: 1437-1443

[98] Dewan A, Srivastava P 2015 A review of heat transfer enhancement through flow disruption in a microchannel. Journal of Thermal Science 24: 203-214

[99] Zhang C, Chen Y and Shi M 2010 Effects of roughness elements on laminar flow and heat transfer in microchannels. Chemical Engineering and Processing 49: 1188-1192

[100] Wei X J, Joshi Y K and Ligrani P M 2007 Numerical simulation of laminar flow and heat transfer inside microchannel with one dimpled surface. ASME Transactions on Journal of Electronic Packaging 129: 63-70

[101] Xu M, Lu H, Gong L, Chai J and Duan X 2016 Parametric numerical study of the flow and heat transfer in microchannel with dimples. International Communications in Heat and Mass Transfer 76: 348-357

[102] Lan J, Xie Y, and Zhang D 2012 Flow and heat transfer in micro channels with dimples and protrusions. ASME Transactions on Journal of Heat Transfer 134: 021901: 1-9

[103] Li P, Xie Y and Zhang D 2016 Laminar flow and forced convective heat transfer of shear-thinning power-law fluids in dimpled and protruded micro channels. International Journal of Heat and Mass Transfer 99: 372-382

[104] Li P, Luo Y, Zhang D and Xie Y 2018 Flow and heat transfer characteristics and optimization study on the watercooled microchannel heat sinks with dimple and pin-fin. International Journal of Heat and Mass Transfer119: $152-162$

[105] Huang X, Yang W, Ming T, Shen W and Yu X 2017 Heat transfer enhancement on a microchannel heat sink with impinging jets and dimples. International Journal of Heat and Mass Transfer 112: 113-124

[106] Al-Asadi M T, Alkasmoul F S and Wilson M C T 2016 Heat transfer enhancement in a micro-channel cooling system using cylindrical vortex generators. International Communications in Heat and Mass Transfer 74: 40-47

[107] Xia G, Jiang J, Wang J, Zhai Y and Ma D 2015 Effects of different geometric structures on fluid flow and heat transfer performance in microchannel heat sinks. International Journal of Heat and Mass Transfer 80: 439-447

[108] Xia G, Chai L, Zhou M and Wang H 2011 Effects of structural parameters on fluid flow and heat transfer in a microchannel with aligned fan-shaped re-entrant cavities. International Journal of Thermal Sciences 50: 411-419

[109] Xia G, Chai L, Wang H, Zhou M and Cui Z 2011Optimum thermal design of microchannel heat sink with triangular reentrant cavities. Applied Thermal Engineering 31: 1208-1219

[110] Chai L, Xia G, Zhou M and Li J 2011 Numerical simulation of fluid flow and heat transfer in a microchannel heat sink with offset fan-shaped reentrant cavities in sidewall. International Communications in Heat and Mass Transfer 38: 577-584.

[111] Kuppusamy N R, Mohammed H and Lim C 2013 Numerical investigation of trapezoidal grooved microchannel heat sink using nanofluids. ThermochimaActa 573: 39-56 
[112] Kuppusamy N R Mohammed H, and Lim C 2014 Thermal and hydraulic characteristics of nanofluid in a triangular grooved microchannel heat sink (TGMCHS).Applied Mathematics and Computation 246: 168-183

[113] Ahmed H and Ahmed M 2015 Optimum thermal design of triangular, trapezoidal and rectangular grooved microchannel heat sinks. International Communications in Heat and Mass Transfer 66: 47-57

[114] Ahmed H, Yusoff M and Shuaib N H 2013 Effects of geometrical parameters on the flow and heat transfer characteristics in trapezoidal-corrugated channel using nanofluid. International Communications in Heat and Mass Transfer 42: 69-74.

[115] Xia G, Zhai Y L and Cui Z 2013 Numerical investigation of thermal enhancement in a micro heat sink with fan-shaped reentrant cavities and internal ribs. Applied Thermal Engineering 58: 52-60.

[116] Zhai Y, Xia G, Liu X and Li Y 2014 Heat transfer in the microchannels with fan-shaped re-entrant cavities and different ribs based on field synergy principle and entropy generation analysis. International Journal of Heat and Mass Transfer 68: 224-233

[117] Bi C, Tang G H and Tao W Q 2013 Heat transfer enhancement in mini-channel heat sinks with dimples and cylindrical grooves. Applied Thermal Engineering 55: 121-132

[118] Chai L, Xia G and Wang H, 2016 Parametric study on thermal and hydraulic characteristics of laminar flow in microchannel heat sink with fan-shaped ribs on sidewalls Part 1: heat transfer, Part 2: pressure drop, Part 3: performance evaluation. International Journal of Heat and Mass Transfer 97:1069-1080, 1081-1090, 1091-1101

[119] Chai L, Xia G and Wang H 2016 Numerical study of laminar flow and heat transfer in microchannel heat sink with offset ribs on sidewalls. Applied Thermal Engineering 92: 32-41

[120] Zhai Y, Xia G, Liu X and Li Y 2015 Exergy analysis and performance evaluation of flow and heat transfer in different micro heat sinks with complex structure. International Journal of Heat and Mass Transfer 84: 293-303

[121] Ghani I A, Sidik N A, Mamat R, Najafi G, Ken T L, Asako Y, and Japar W M 2017 Heat transfer enhancement in microchannel heat sink using hybrid technique of ribs and secondary channels. International Journal of Heat and Mass Transfer 114: 640-55

[122] Xia G, Ma D, Zhai Y, Li Y, Liu R and Du M 2015 Experimental and numerical study of fluid flow and heat transfer characteristics in microchannel heat sink with complex structure. Energy Conversion and Management 105: 848-857

[123] Li Y, Xia G, Ma D, Jia Y and Wang J 2016 Characteristics of laminar flow and heat transfer in microchannel heat sink with triangular cavities and rectangular ribs. International Journal of Heat and Mass Transfer 98: 17-28

[124] Datta A, Sharma V, Sanyal D and Das P 2019 A conjugate heat transfer analysis of performance for rectangular microchannel with trapezoidal cavities and ribs. International Journal of Thermal Sciences 138: 425-446

[125] Foong A, Ramesh N and Chandratilleke T 2009 Laminar convective heat transfer in a microchannel with internal longitudinal fins. International Journal of Thermal Sciences 48: 1908-1913

[126] Xie G, Shen Hand Wang C 2015 Parametric study on thermal performance of microchannel heat sinks with internal vertical Y-shaped bifurcations. International Journal of Heat and Mass Transfer 90: 948-958

[127] Xie G, Zhang F, Sunden B and Zhang W 2014Constructal design and thermal analysis of microchannel heat sinks with multistage bifurcations in single-phase liquid flow. Applied Thermal Engineering 62: 791-802

[128] Jia Y, Xia G, Li Y, Ma D and Cai B 2018 Heat transfer and fluid flow characteristics of combined microchannel with cone-shaped micro pin fins. International Communications in Heat and Mass Transfer 92: 78-89

[129] Colgan E G, Furman B, Gaynes M, Graham W S, LaBianca N C, Magerlein J H, Polastre RJ, Rothwell MB, Bezama RJ, Choudhary R, Marston K C, Toy H, Wakil J, Zitz J A and Schmidt RR 2007 A practical implementation of silicon microchannel coolers for high power chips. IEEE Trans on Component Packaging and Technologies 30: 218-225

[130] Hong F, and Cheng P 2009 Three dimensional numerical analyses and optimization of offset strip-fin microchannel heat sinks. International Communications in Heat and Mass Transfer 36: 651-656

[131] Lee Y, Lee P and Chou 2014 Enhanced thermal transport in microchannel using oblique fins. Trans ASME Journal of Heat Transfer 134 (10): 101901:1-10

[132] Kuppusamy N R, Saidur R, Ghazali N and Mohammed H 2014 Numerical study ofthermal enhancement in micro channel heat sink with secondary flow. International Journal of Heat Mass Transfer78: 216-223

[133] Kandlikar S G, Garimella S, Li D, Colin S and King M R Heat Transfer and Fluid Flow in Minichannels and Microchannels. Elsevier Science Ltd, Singapore

[134] Xu J, Gan Y H, Zhang D C and Li X 2005 Micro scale heat transfer enhancement using thermal boundary layer redeveloping concept. International Journal of Heat Mass Transfer 48: 1662-1674

[135] Chai L, Xia G, Zhou M, Li J and Qi J 2013 Optimum thermal design of interrupted microchannel heat sink with rectangular ribs in the transverse microchambers. Applied Thermal Engineering 51: 880-889

[136] Chai L, Xia G D and Wang H S 2016. Laminar flow and heat transfer characteristics of interrupted microchannel heat sink with ribs in the transverse microchambers. International Journal of Thermal Sciences 110: 1-11

[137] Chandratilleke T T, Jagannatha D and Narayanaswamy R, 2010, Heat transfer enhancement in micro channels with cross-flow synthetic jets. International Journal of Thermal Sciences 49: 504-513

[138] Lee A, Timchenko V, Yeoh G H and Reizes J A 2012 Three-dimensional modeling of fluid flow and heat transfer in micro-channels with synthetic jet. International Journal of Heat and Mass Transfer 55: 198-213

[139] Lee A, Timchenko V, Yeoh G H and Reizes J A 2012 Heat transfer enhancement in micro-channel with multiple synthetic jets. Applied Thermal Engineering 48: 275-288

[140] Fang R and Khan J A 2013 Active Heat Transfer Enhancement in Single-Phase Micro channels by using Synthetic Jets. Trans ASME Experimental Journal of 
Thermal Science and Engineering Applications 5: 011006:1-8

[141] Kandlikar S G and Bapat A V 2007. Evaluation of jet impingement, spray and microchannel chip cooling options for high heat flux removal. Heat Transfer Engineering 28: 911-923

[142] Meis M, Varas F, Velázquez A and Vega J M 2010 Heat transfer enhancement in micro-channels caused by vortex promoters. International Journal of Heat and Mass Transfer 53: 29-40

[143] Koz M, Ozdemir M R and Kosar A 2011 Parametric study on the effect of end walls on heat transfer and fluid flow across a micro pin-fin. International Journal of Thermal Sciences 50: 1073-1084

[144] Wang Q, Chen Q, Wang L, Zeng M, Huang Y and Xio Z 2007 Experimental study of heat transfer enhancement in narrow rectangular channel with longitudinal vortex generators. Nuclear Engineering and Design 237: 686-93

[145] Wu J and Tao W 2008 Numerical study on laminar convection heat transfer in a rectangular channel with longitudinal vortex generator, Part A: Verification of field synergy principle, Part B: Parametric study of major influence factors. International Journal of Heat and Mass Transfer 51: 1179-1191, 3683-3692

[146] Hsiao K, Wu C and Huang Y 2014 Fluid mixing in a microchannel with longitudinal vortex generators. Chemical Engineering Journal 235: 27-36

[147] Liu C, Teng J, Chu J, Chiu Y, Huang S, Jin S, Dang T, Greif R and Pan H 2011 Experimental investigations on liquid flow and heat transfer in rectangular micro channel with longitudinal vortex generators. International Journal of Heat and Mass Transfer 54: 3069-3080

[148] Chen C, Teng J, Cheng C, Jin S, Huang S, Liu C, Lee M, Pan H, and Greif R 2014 A study on fluid flow and heat transfer in rectangular microchannels with various longitudinal vortex generators. International Journal of Heat and Mass Transfer 69: 203-214

[149] Ebrahimi A, Roohi E and Kheradmand S 2015 Numerical study of liquid flow and heat transfer in rectangular microchannel with longitudinal vortex generators. Applied Thermal Engineering 78: 576-583

[150] Datta A, Sanyal D and Das A 2016 Numerical investigation of heat transfer in microchannel using inclined longitudinal vortex generator. Applied Thermal Engineering 108: 1008-1019

[151] Steinke M E and Kandlikar S G 2004 Single-phase heat transfer enhancement techniques in microchannel and minichannel flows. In: ASME 2004 2nd International Conference on Microchannels and Minichannels, pp. $141-148$

[152] Chu J, Teng J and Greif R 2010 Experimental and numerical study on the flow characteristics in curved rectangular microchannels. Applied Thermal Engineering 30: $1558-1566$

[153] Chu J, Teng J, Xu T, Huang S, Jin S, Yu X, Dang T, Zhang $\mathrm{C}$ and Greif R 2012 Characterization of frictional pressure drop of liquid flow through curved rectangular microchannels. Experimental Thermal and Fluid Science 38: 171-183

[154] Yang W, Zhang J Z and Cheng H 2005 The study of flow characteristics of curved microchannel. Applied Thermal Engineering 25: 1894-1907
[155] Sui Y, Teo C J and Lee P S 2011 An experimental study of flow friction and heat transfer in wavy microchannels with rectangular cross-section. International Journal of Thermal Sciences 50: 2473-2482

[156] Sui Y, Teo C J, Lee P S, Chew Y T and Shu C 2010 Fluid flow and heat transfer in wavy microchannels. International Journal of Heat and Mass Transfer 53: 2760-2772

[157] Duryodhan V S, Singh A, Singh S G and Agrawal A 2015 Convective heat transfer in diverging and converging microchannels. International Journal of Heat and Mass Transfer 80: 424-438

[158] Duryodhan V S, Singh S G and Agrawal A 2013 Liquid flow through a diverging microchannel. Microfluidics and Nanofluidics 14: 53-67

[159] Duryodhan V S, Singh S G and Agrawal A 2014 Liquid flow through converging microchannel and comparison with diverging microchannel. Journal of Micromechanics and Microengineering 24: 125002:1-13

[160] Gong L, Kota K, Tao W and Joshi Y 2011 Parametric numerical study of flow and heat transfer in microchannels with wavy walls. Trans ASME Journal of Heat Transfer 133: 051702:1-10

[161] Ghaedamini H, Lee P S and Teo C J 2013 Developing forced convection in converging-diverging microchannels. International Journal of Heat and Mass Transfer 65: 491-499

[162] Yong J and Teo C J 2014 Mixing and heat transfer enhancement in microchannels containing convergingdiverging passages. Trans ASME Journal of Heat Transfer 136: 041704:1-11

[163] Dharaiya V V and Kandlikar S G 2013 A numerical study on the effects of $2 \mathrm{~d}$ structured sinusoidal elements on fluid flow and heat transfer at microscale. International Journal of Heat and Mass Transfer 57: 190-201

[164] Ma D D, Xia G, Li Y, Jia Y and Wang J 2011 Effects of structural parameters on fluid flow and heat transfer characteristics in microchannel with offset zigzag grooves in sidewall. International Journal of Heat Mass Transfer 101: 427-435

[165] Mohammed H A, Gunnasegaran P and Shuaib N H 2011 Numerical simulation of heat transfer enhancement in wavy microchannel heat sink. International Communications in Heat and Mass Transfer 38: 63-68

[166] Alam A and Kim K Y 2012 Analysis of mixing in a curved microchannel with rectangular grooves. Chemical Engineering Journal 181-182: 708-716

[167] Sakanova A, Keian C C and Zhao J 2015 Performance improvements of microchannel heat sink using wavy channel and nanofluids. International Journal of Heat and Mass Transfer 89: 59-74

[168] Chiam Z L, Lee P S, Singh P K, and Mou N 2016 Investigation of fluid flow and heat transfer in wavy microchannels with alternating secondary branches. International Journal of Heat and Mass Transfer 101: 1316-1330

[169] Aref H 1984 Stirring by chaotic advection. Journal of Fluid Mechanics 143: 1-21.Aref

[170] Aref H 2002 The development of chaotic advection. Physics of Fluids 14: 1315-1325

[171] Ottino J M 1989 The kinematic of mixing: stretching, chaos and transport. New York: Cambridge University Press 
[172] Stroock A D, Dertinger S K W, Ajdari A, Mezic I, Stone H A and Whitesides G M 2002 Chaotic mixer for microchannels. Science 295: 647-651

[173] Schönfeld F and Hardt S 2004, Simulation of helical flows in micro channels. AIChE Journal 50: 771-778

[174] Jiang F, Drese K S, Hardt S, Küpper M and Schönfeld F 2004 Helical flows and chaotic mixing in curved microchannels. AIChE Journal 50: 2297-2305

[175] Kalb C E and Seader J D 1972 Heat and mass transfer phenomena for viscous flow incurved circular tubes. International Journal of Heat and Mass Transfer 15: 801-817

[176] Masliyah J H and Nandakumar K 1979 Fully-developed viscous flow and heat transfer in curved semi-circular sectors. AIChE Journal 25 (3): 478-487

[177] Yang G, Dong Z F and Ebadian M A 1995 Laminar forced convection in a helicoidal pipe with fine pitch. International Journal of Heat and Mass Transfer 38 (5): 853-862

[178] Wang L and Yang T 2004 Bifurcation and stability of forced convection in curved ducts of square cross-section. International Journal of Heat and Mass Transfer 47: 2971-2987

[179] Sui Y, Teo C J and Lee P S 2012 Direct numerical simulation of fluid flow and heat transfer in periodic wavy channels with rectangular cross-sections. International Journal of Heat and Mass Transfer 55: 73-88

[180] Zheng Z, Fletcher C F and Haynes B S 2013 Laminar heat transfer simulations for periodic zigzag semicircular channels: Chaotic advection and geometric effects. International Journal of Heat and Mass Transfer 62: 391-401

[181] Abed W M, Whalley R D, Dennis D J C and Poole R J 2015 Numerical and experimental investigation of heat transfer and fluid flow characteristics in a micro-scale serpentine channel. International Journal of Heat and Mass Transfer 88: $790-802$

[182] Dean W R 1927 XVI. Note on the motion of fluid in a curved pipe. London, Edinburgh, Dublin Philosophical Magazine and Journal of Science 4: 208-223

[183] Dean W R, 1928 LXXII. The stream-line motion of fluid in a curved pipe (Second paper). London, Edinburgh, Dublin Philosophical Magazine and Journal of Science 5: 673-695

[184] Sugiyama S, Hayashi T, and Yamazaki K 1983 Flow characteristics in the curved rectangular channels: visualization of secondary flow. Bulletin JSME 26: 964-969

[185] Mokrani A, Castelain C and Peerhossaini H 1997 The effects of chaotic advection on heat transfer. International Journal of Heat and Mass Transfer 40: 3089-3104

[186] Xie G, Liu J, Zhang W and Sunden B 2012 Analysis of flow and thermal performance of a water-cooled transversal wavy microchannel heat sink for chip cooling. Trans. ASME Journal of Electronic Packaging 134: 021008:1-11

[187] Xie G, Liu J, Liu Y, Sunden B and Zhang W 2013 Comparative study of thermal performance of longitudinal and transversal-wavy microchannel heat sinks for electronic cooling. Trans. ASME Journal of Electronic Packaging 135: $1-9$

[188] Facao J and Oliveira A C 2005 Modelling laminar heat transfer in a curved rectangular duct with a computational fluid dynamics code. Numerical Heat Transfer Part A 48: $165-177$

[189] Rosaguti N R, Fletcher D F and Haynes B S 2005 Laminar flow and heat transfer in a periodic serpentine channel. Chemical Engineering Technology 28: 353-361

[190] Rosaguti N R, Fletcher D F and Haynes B S 2006 Laminar flow and heat transfer in a periodic serpentine channel with semi-circular cross-section. International Journal of Heat and Mass Transfer 49: 2912-2923

[191] Rosaguti N R, Fletcher D F and Haynes B S 2007 LowReynolds number heat transfer enhancement in sinusoidal channels, Chemical Engineering Science 62: 694-702

[192] Geyer P E, Rosaguti N R, Fletcher D F and Haynes B S 2006 Thermohydraulics of square-section microchannels following a serpentine path. Microfluidics and Nanofluidics 2: 195-204

[193] Wang L and Liu F 2007 Forced convection in slightly curved microchannels. International Journal of Heat and Mass Transfer 50: 881-896

[194] Dai Z, Fletcher C F and Haynes B S 2015 Impact of tortuous geometry on laminar flow heat transfer in microchannels. International Journal of Heat and Mass Transfer 83: 382-398

[195] Dai Z, Zheng Z, Fletcher C F and Haynes B S 2015 Experimental study of transient behaviour of laminar flow in zigzag semi-circular microchannels. Experimental Thermal and Fluid Science 68: 644-651

[196] Hasis F B, Krishna P M, Aravind G P, Deepu M and Shine S R 2018 Thermo hydraulic performance analysis of twisted sinusoidal wavy microchannels. International Journal of Thermal Sciences 128: 124-36

[197] Datta A, Das A K, Dey P and Sanyal D 2017 Multiobjective optimization of laminar heat transfer and friction factor in rectangular microchannel with rectangular vortex generators: an application of NSGA-II with gene expression programing metamodel. Journal of Heat Transfer 139: 072401.

[198] Wong K and Lee J 2015 Investigation of thermal performance of microchannel heat sink with triangular ribs in the transverse micro chambers. International Communications in Heat and Mass Transfer 65: 103-110

[199] Shen H, Wang C C and Xie G 2018 A parametric study on thermal performance of microchannel heat sinks with internally vertical bifurcations in laminar liquid flow. International Journal of Heat and Mass Transfer117: 487-497

[200] Peng XF, Peterson G P and Wang B X 1995 Heat transfer characteristics of water flowing through microchannels. Journal Experimental Heat Transfer 7: 265-283 